Document downloaded from:

http://hdl.handle.net/10251/121370

This paper must be cited as:

Boronat, F.; Montagud, M.; Marfil-Reguero, D.; Luzón, C. (2018). Hybrid Broadcast/Broadband TV Services and Media Synchronization. Demands, Preferences and Expectations of Spanish Consumers. IEEE Transactions on Broadcasting. 64(1):52-69. https://doi.org/10.1109/TBC.2017.2737819

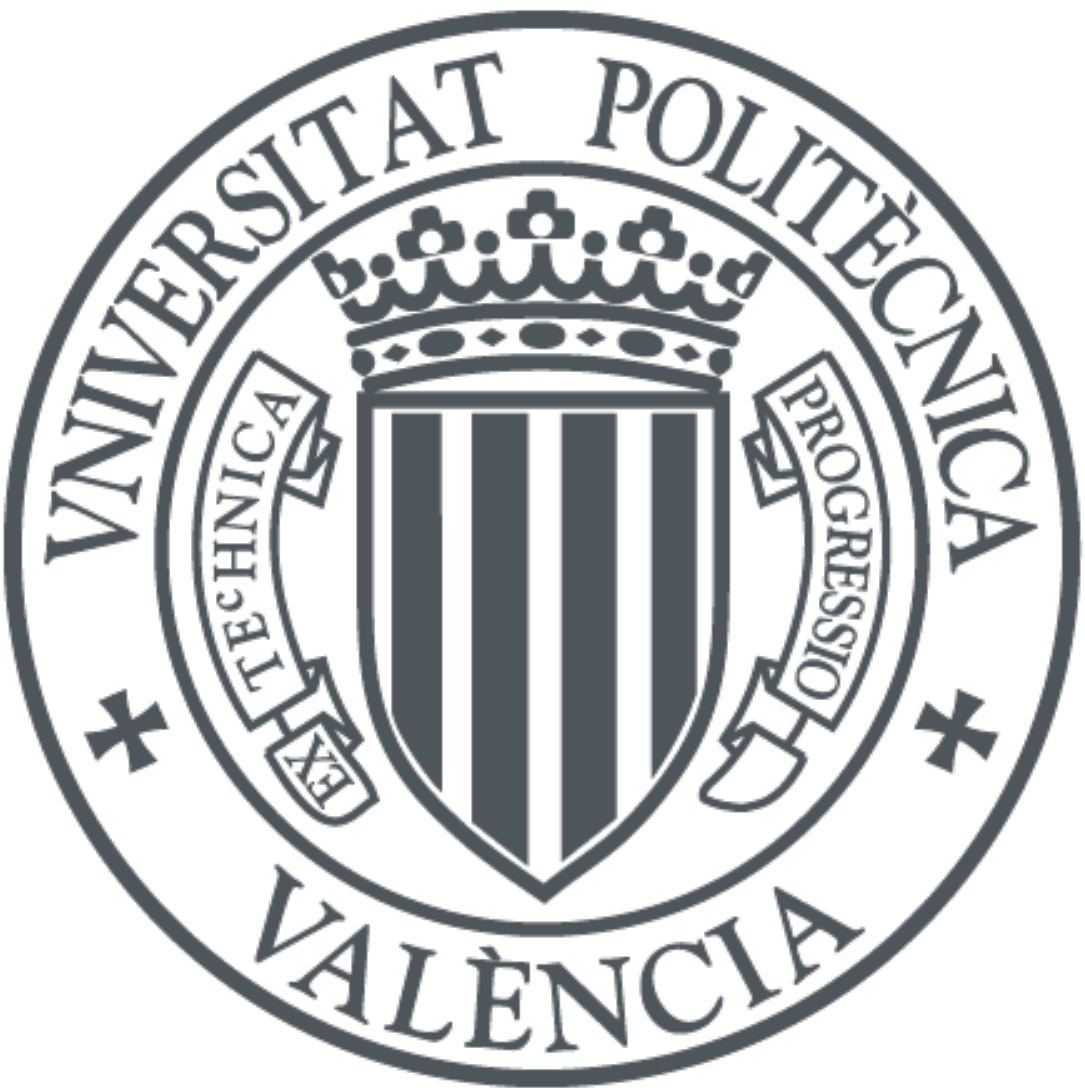

The final publication is available at

http://doi.org/10.1109/TBC.2017.2737819

Copyright Institute of Electrical and Electronics Engineers

Additional Information 


\title{
Hybrid Broadcast/Broadband TV Services and Media Synchronization. Demands, Preferences and Expectations of Spanish Consumers
}

\author{
Fernando Boronat, Senior, IEEE, Mario Montagud, Member, IEEE, Dani Marfil and Clara Luzón
}

\begin{abstract}
The combination of broadcast and broadband (hybrid) technologies for delivering TV related media contents is already a reality. It has been motivated by the large amount and diversity of media contents, together with the ubiquity and multiple connectivity capabilities of modern consumption devices. The use of connected TVs and companion devices (e.g., tablets, smartphones...) is gaining momentum. It enables personalized and enriched TV media experiences, by also exploiting social communication opportunities. Likewise, the media consumption paradigm is worldwide evolving from passive and isolated consumer experiences towards interactive and group shared experiences between remote consumers. Nevertheless, despite the specification of standards, such as Hybrid Broadcast Broadband TV (HbbTV), and the efforts from operators and content providers in the last years, the adoption of hybrid TV media services in Europe is still not as high as expected. This paper presents the concept and some examples of hybrid TV media services, emphasizing the importance of including a combination of media synchronization solutions, known as hybrid sync, to guarantee a satisfactory level of Quality of Experience (QoE). Additionally, it includes the summary and discussion of the results of a research study focused on more than 1000 Spanish users' habits, preferences and expectations regarding four representative hybrid TV media services. Many valuable insights and conclusions have been derived. For instance, the current low market adoption of hybrid TV media services, despite their advantages and the high interest of consumers, and that key technological challenges still need to be overcome. The obtained results and impressions foresee the impact (or potential) of such services in the upcoming TV related media consumption landscape. Therefore, devising proper standard-compliant technological solutions (paying special attention to hybrid sync) and equipment should be continued producing appropriate contents, deploying proper hybrid TV media services and applications. As well, it should be convenient to undertake marketing and commercial efforts to boost their deployment. The contributions of this study can be very valuable to the interested agents to be aware of the remaining challenges, envisage the opportunities, and drive their efforts to maximize the market adoption of such services.
\end{abstract}

Index Terms - Broadcast technology, Digital multimedia broadcasting, TV broadcasting, HbbTV, Connected TV, Interactive TV, Social TV, media synchronization, QoE.

Submited: February 7, 2017.

"This work has been funded, partially, by the "Fondo Europeo de Desarrollo Regional (FEDER)" and the Spanish Ministry of Economy and Competitiveness (MINECO), under its R\&D\&I Support Program, in project with Ref. TEC2013-45492-R.

\section{INTRODUCTION}

CURRENTLY, the large availability and diversity of media contents, together with the ubiquity and the multiple connectivity capabilities of modern consumption devices, already enable the combination and coordination of broadcast/broadband (hybrid) delivery technologies for media consumption. According to studies in [1] and [2], Future Internet will carry high-quality heterogeneous (hybrid) media contents and services, integrating social communications with other interactive applications. This heterogeneous media ecosystem may indeed lead to rivalry and incompatibility problems. However, it also brings new fascinating research and development opportunities.

As a parallel phenomenon, the media consumption paradigm is worldwide evolving from isolated and passive consumer experiences towards group shared and interactive experiences between remote consumers. An important goal for nextgeneration TV media services is to efficiently support shared experiences. In such experiences, groups of consumers will be able to interact and share services independently of their location, access network and consumption devices. A typical example is Social TV, in which a group of geographically distributed consumers (e.g., friends or relatives) is watching TV together, while socially interacting via text chat or audio/video conferencing tools (a.k.a. Watching Apart Together case). This emerging paradigm is driven by the humans' natural desire, as being social species, to share information and to consume content as part of a group [3].

In these increasingly relevant scenarios, the use of Smart TVs and companion devices (e.g., tablets, smartphones...) is gaining momentum, enabling personalized and enriched media experiences. A new wave of enriched services, and even new business models, are now possible. This is particularly relevant to TV operators and other stakeholders (e.g., device manufacturers, content providers...). The (linear) broadcast TV content can be augmented by on-demand media content delivered via broadband technologies. Nevertheless, despite the efforts from some initiatives to drive hybrid TV media services,

Authors are with the Universitat Politecnica de Valencia - Campus de Gandia, Immersive and Interactive R\&D group, Communications Department, 46730, Grao de Gandia, Valencia, SPAIN (e-mails: \{fboronat@dcom, mamontor@,damarre@dcom, clalual@epsg\}.upv.es). 
such as HbbTV (Hybrid Broadcast Broadband TV) [4], ATSC (Advanced Television Systems Committee) [5] and MMT (MPEG Media Transport) [6] standards and the Smart TV Alliance $^{1}$, the current solutions still do not fully exploit the potential and opportunities that these services can offer.

Further research is still needed to guarantee a satisfactory Quality of Experience (QoE) in such services. Overcoming remaining challenges, such as dynamic community building, media synchronization (sync hereafter), Quality of Service (QoS), scalability, presence awareness, privacy concerns, and social networking integration [7] is paramount. Among them, as recently highlighted in the last HbbTV Symposium ${ }^{2}$, hybrid sync is still an unsolved problem in such services. The hybrid sync term is used when different related streams, delivered via hybrid broadcast/broadband technologies, need to be played out in a synchronous way. It can involve one or several well-known media sync types (explained in Section III) to guarantee an insync playout of these related streams. Timing artifacts introduced by the agents in the end-to-end multimedia distribution chain must be corrected at the destination/s to provide in-sync presentation/s and good QoE levels.

Previous studies, e.g. [8] and [9], have analyzed the new trends in the media consumption landscape. Both of them show a rise in the use of multiple-screen apps (e.g., to connect to social networks and/or browse while watching TV). Other studies have even compared that increase with the rise of using Smart TV for those tasks ${ }^{3}$. Nevertheless, no studies focused on analyzing the consumer's habits, demands, opinions, preferences and expectations when consuming hybrid TV related media contents have been found. This has been the main motivation for conducting such a research study focused on our own country (Spain). It has addressed the Spanish consumers' point of view and four relevant hybrid media consumption services (described in Section IV.A).

In this paper, first, the concept of hybrid TV media services, together with some illustrative examples, are presented. The need for different types of media sync (as it is a key technological enabler for these services) in such services is highlighted. Then, the results of the aforementioned study are presented and discussed. The results reveal many valuable insights, such as the low market adoption of hybrid TV media services, despite their advantages, and the high interest of consumers. Nevertheless, further research is needed to overcome key remaining challenges, with a special focus on hybrid sync.

Although the study has been solely conducted for Spanish consumers, Spain is one of the main European countries in which hybrid TV media services (based on Smart TVs and especially on the HbbTV standard) are regularly operating and being progressively adopted and improved. Accordingly, the obtained results and conclusions are not only relevant for Spanish, but also for other international stakeholders in which similar initiatives to drive hybrid TV media services are being

\footnotetext{
${ }^{1}$ http://smarttv-alliance.org/

http://hbbtvsymposium.evolero.com/2016/

http://advanced-television.com/2015/01/15/industry-pros-favour-2ndscreen-over-smart-tv/ (last access: January 2016).
}

carried out. The contributions of this paper can be very valuable for the involved agents in the worldwide media consumption landscape to envisage what still needs to be developed, offered and improved in next-generation hybrid TV media services.

The structure of the paper is as follows. The concept of hybrid TV media services is introduced in Section II, including some illustrative examples. Then, in Section III, the delay variability problem in hybrid media scenarios is presented, and the need for hybrid sync is justified. In Section IV, the research study focused on Spanish consumers is presented. The cases of usage by the participants were asked about, the employed methodology, the sample of participants, general data, and a summary of the obtained results are included. These results are then discussed in Section V. Finally, the paper ends with some conclusions in Section VI.

\section{Hybrid TV MEdiA TeChNOLOGIES AND SERVICES}

A huge variety of delivery technologies, consumption devices and media contents are currently at the consumers' disposal. Regarding distribution, media can be delivered via broadcast and broadband technologies. On the one hand, broadcast technologies, such as Digital Video Broadcasting (DVB), can concurrently deliver the same media content to a large number of consumers. In this context, media can be broadcast by using terrestrial (e.g., DVB-T), satellite (e.g., DVB-S), mobile (e.g., DVB-H) and cable (e.g., DVB-C) technologies. On the other hand, broadband IP technologies can provide interactive, bidirectional and adaptive services, tailored to the resources and/or preferences of the customers. However, they typically provide poorer performance in terms of scalability, stability and latency compared to broadcast technologies. In this context, media can be delivered by using different forms of IP-based downloading and streaming techniques, of which the latter are becoming more popular.

Two main types of streaming media services can be distinguished: managed and unmanaged. On the one hand, managed services typically operate within (controlled) walledgarden environments (e.g., Internet Protocol Television or IPTV). They mainly rely on push-based streaming, by making use of the standard protocols Real Time Transport Protocol (RTP) and its companion RTP Control Protocol (RTCP) [10]. They are especially suited for delay-sensitive and interactive services. On the other hand, unmanaged services can operate worldwide, and mainly rely on pull-based HTTP Adaptive Streaming (HAS) solutions. Their main advantages are adaptability, scalability, reliability, ubiquity and cost efficiency. In this context, different vendors and standardization bodies have specified their own HAS solution, such as HTTP Live Streaming ${ }^{4}$ (HLS) by Apple, Dynamic Adaptive Streaming over HTTP ${ }^{5}$ (DASH) by ISO/IEC and MPEG, HTTP Dynamic Streaming ${ }^{6}$ (HDS) by Adobe, and Microsoft Smooth Streaming ${ }^{7}$ (MSS) by Microsoft. HAS solutions are under unceasing improvement and are being increasingly adopted for broadband media delivery (e.g., DASH has been adopted by the

\footnotetext{
${ }^{4}$ https://tools.ietf.org/html/draft-pantos-http-live-streaming-20

5 http://mpeg.chiariglione.org/standards/mpeg-dash

${ }^{6} \mathrm{http} / / /$ www.adobe.com/products/hds-dynamic-streaming.html

${ }^{7}$ https://www.iis.net/downloads/microsoft/smooth-streaming
} 
HbbTV standard [4], and is employed in many popular media services, such as Netflix and YouTube).

Regarding consumption devices, apart from the wide availability of connected (i.e., IP-enabled) or Smart TVs, the proliferation and massive usage of different kinds of companion (a.k.a. secondary) devices are a reality. These devices are equipped with multiple connectivity capabilities (WiFi, 3G/4G/5G, FM...), which enable the concurrent consumption of different media contents via the same or different delivery technologies. They can have heterogeneous resources, performance and capabilities (e.g., available bandwidth, supported technologies and media types, media processing capabilities and resolution displays).

In this paper, the hybrid TV media services concept is also introduced. It refers to those interactive media services that concurrently make use of broadcast and broadband technologies to consume TV-related media contents. These contents can be consumed on just one device (a.k.a. hybrid terminal, e.g. a connected TV) or on several devices in multiscreen scenarios (e.g., one or more hybrid terminals and/or one or more companion devices).

Figure 1 shows the main concept of a hybrid TV media system. It is the system needed to support hybrid TV media services. MS stands for Main Screen and CS for Companion Screen functionalities, according to the terminology of the HbbTV standard.

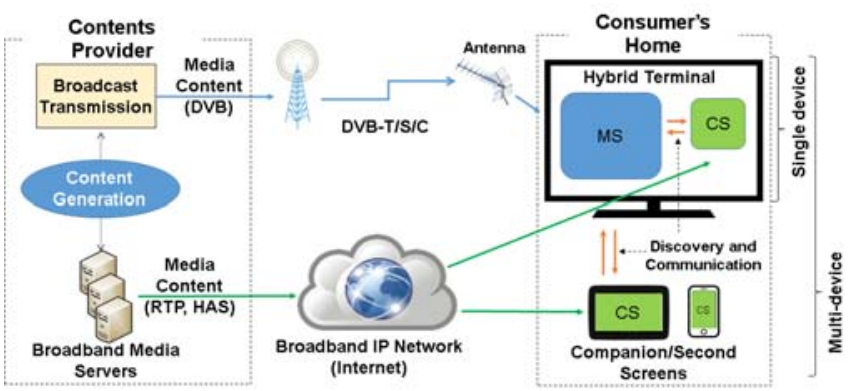

Fig. 1. Overall Hybrid TV media system

Hybrid TV media services have caught the attention of the scientific community and public organizations in recent times. Various related projects financed by the EU in the last years, such as HBB-NEXT ${ }^{8}$ (Next-Generation Hybrid Broadcast Broadband), HBB4ALL ${ }^{9}$ (Hybrid Broadcast Broadband for All) or TV-RING ${ }^{10}$, among others, have been focused on the use of hybrid technologies to offer enhanced TV services, mainly based on the HbbTV standard. Many of the research studies referenced in this paper come from contributions achieved under the umbrella of these projects. The HBB-NEXT project contributed to improve the first version of the HbbTV standard (some of the proposals have been included in the latest version of the standard, HbbTV 2.0.1) and with tools for HbbTV-based apps development. HBB4ALL addressed global media accessibility issues in these heterogeneous and connected TV environments. The deployment of customizable accessible TV services (e.g., including videos with sign language, or adaptive subtitles), according to the needs or preferences of the consumers, has been one of the goals of this project. The objective of the TV-RING project was to develop and test applications and infrastructures shaping Internet-based TV, aiming to deliver high quality services and offer innovative contents on the top of traditional broadcasting.

\section{A. Examples of Hybrid TV Media Services}

Many hybrid TV media services and consumption scenarios can be provided. Next, some relevant examples are listed:

a) Provision of additional audio streams via broadband technologies. For example, it allows the substitution of the built-in audio channel from the broadcast stream by the one from the favorite on-line radio channel or in the preferred language (customized selection of audio streams).

b) Multi-view (or Free ViewPoint) TV. It allows providing different views of an event. With just one view being broadcast and the rest being delivered via broadband technologies, this avoids requiring extra broadcast resources.

c) Remote Participation in TV shows. For example, users can virtually and remotely participate in TV shows/programs, independently from their location. Starting from simple messaging (e.g., for commenting or voting purposes), they can become live participants in TV shows, even with live streaming footage through webcams and real-time interaction. In quiz TV shows, they could participate and answer the questions on their own Smart TV or on companion devices, and send the answers through broadband technologies.

d) Social TV (a.k.a. Watching Apart Together). It allows augmenting the broadcast TV content with interactive (text and/or audiovisual) communication channels, so that remote users can socially interact within the context of the same TV content. A common example is a group of friends watching an online football match, each one from a different location, while discussing the unfolding of the match and sharing emotions (e.g., cheering together).

e) Provision of spatial, temporal and color scalability via broadband to augment the quality of the broadcast TV contents.

f) Tiled streaming. It consists of ultra-high resolution video services in which different spatial areas (tiles) of the same scene or event are delivered in different video streams. In this case, for example, the view of the overall area can be delivered via broadcast, and the views of the other areas via broadband.

g) Provision of advanced functionalities (e.g., catch-up, replays, fast rewind/forward...).

h) (Targeted) Commercials. Consumers can be provided with targeted commercials. They can be based on their profiles, interests, or even on their activity during the TV experience.

i) Integration of web feeds (e.g., including quizzes, relevant information, statistics...).

This enriched TV media consumption paradigm and new hybrid TV media services, on the one hand, have a big impact for entertainment purposes. On the other hand, they can also bring social benefits, so that users can feel more integrated and immersed when consuming media. To name a few examples, we can find native audio language selection, inclusion of videos 
with sign language and customized/adaptive presentation of subtitles or audio descriptions. These can be very useful and valuable, especially for elderly or disabled consumers.

It could be technically feasible to provide most of the possible augmented services using exclusively broadcast technologies. Nevertheless, it must be taken into account that all of them require additional bandwidth (a scarce and expensive asset in the broadcast domain), and that not all the customers may want to pay for (or even -freely- use) them. Therefore, the use of broadband delivery technologies to provide these augmented services only to those interested customers is a more efficient strategy, reducing costs.

\section{Delay Variability: NeEd for Hybrid SyNC}

Hybrid sync is a key challenge to tackle in hybrid TV media services. Delay and its variability when delivering one or more media streams to one or more receivers through hybrid delivery technologies is a barrier for deploying such services. Timing artifacts introduced by the agents in the end-to-end multimedia delivery chain will result in time lags (within each individual stream, between media streams and between receivers). Various system components and factors along the chain (can) contribute to the increase of delays and of their variability, having a significant impact on media sync, as illustrated in Figure 2. This figure shows the difference of the playout delays (i.e., asynchrony) between two streams (streams 1 and 2) generated at the same time. It is mainly due to the existence of various factors, some of which occur in different stages of the media delivery process: the media source or technology, the distribution network and/or the consumer devices. Capturing, encryption (if used), coding, packetization, distribution (including traffic load, packet losses, trans-coding or format conversion -if needed-, fragmentation and re-assembly of packets, multicast or dynamic routing strategies, improper queuing policies...), and the reverse tasks at the consumer's side can seriously disturb the original media timing. The existence of all these factors can result in different time variant end-toend delays when delivering media content from one or multiple media sources to one or multiple receivers/devices, when using either the same or different delivery technologies. For example, Figure 2 also shows a delay variability for the same media stream (stream 1) when it is simultaneously delivered via different technologies (technologies 1 -left side- and 3 -right side-).

These delay differences (and variations) have to be corrected at the consumption device/s in order to provide in-sync playout processes for all the involved related streams, regardless of the delivery technology in use. The use of a combination of different types of media sync solutions will be needed, depending on the specific scenario [1]: the sync of the media playout process for each individual media stream (a.k.a. intrastream sync); and the sync between the playout processes of related streams (a.k.a. inter-stream sync). Those streams can be played out on the same hybrid device (e.g., a Smart TV) or on different devices, which, in turn, can be either close-by (a.k.a. inter-device sync or IDES) or far apart, i.e. in different locations (a.k.a. inter-destination media sync, IDMS). More detailed definitions and examples of all these kinds of media sync types and examples can be found in [1] and [11].

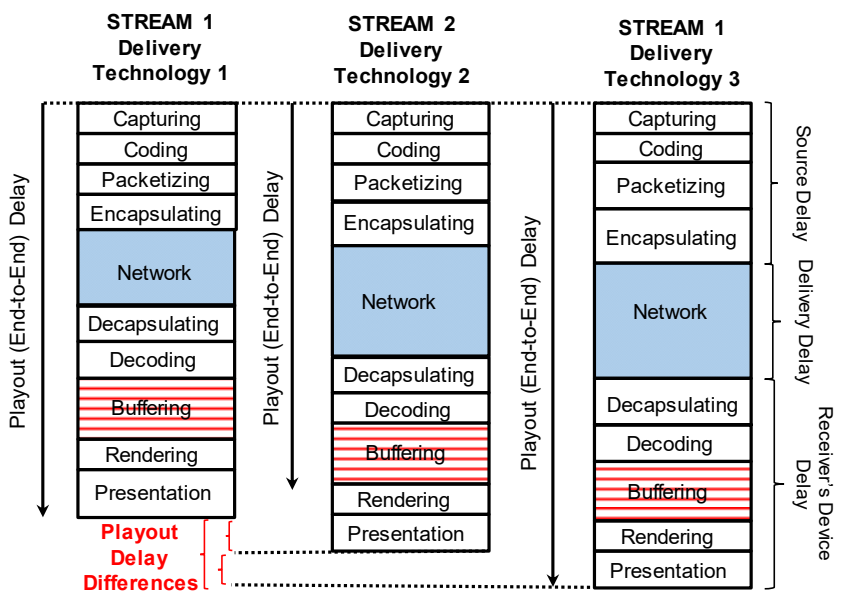

Fig. 2. End-to-end delay variability: Need for Hybrid Sync

Some previous works have measured and/or reported on the magnitudes of delays and their variability when delivering media contents via broadcast and via broadband. On the one hand, different TV broadcasting technologies make use of different distribution channels and techniques. These differences also imply a variability of end-to-end delays between them. For example, the measurements presented in [12], [13] and [14] revealed that the end-to-end delay differences when receiving the same media content (in the same or different format) via different TV broadcast technologies can accumulate up to $5 \mathrm{~s}$. Similarly, the study in [15] provided measurements about the magnitudes of delay differences for different TV setups in specific receivers. It was shown that delay differences between different TV broadcasts in a national scenario (in the Netherlands) can accumulate up to almost $5 \mathrm{~s}$, while in an international scenario (between the Netherlands and the UK) can accumulate up to $6 \mathrm{~s}$. Likewise, these measurements revealed that analog broadcast contents are typically delivered faster than digital broadcast ones (the latter involve extra processes, such as encoding or transcoding, which contribute to increase the delay). Moreover, usually the broadcast delivery of High Definition (HD) contents is slightly slower than the broadcast delivery of Standard Definition (SD) contents.

Furthermore, the broadcast technology used for delivery has a clear influence on the end-to-end delays. For example, satellite communications (DVB-S) involve an extra delay of at least some hundreds of ms [16]. It is mainly due to the delay introduced by the signal propagation process to and from the satellite at the speed of light. Other broadcasting technologies, such as cable TV (DVB-C) or terrestrial TV (DVB-T), have a much smaller and lower boundary of the minimum transmission delay.

On the other hand, regarding broadband delivery technologies, the International Telecommunications Union (ITU-T) G.1050 standard [17] reports on typical values of delays and jitter (delay variability) in Internet. It states that network delays typically range between 20 and $500 \mathrm{~ms}$, while jitter values range between 0 and $500 \mathrm{~ms}$. Likewise, the ITU-T G.114 standard [18] indicates that delays lower than $150 \mathrm{~ms}$ are required for Internet conferencing, while delays larger than $400 \mathrm{~ms}$ are typically unacceptable. In [19], it is reported that the end-to-end delays when using different videoconferencing 
systems can range between a few tens of ms to more than $300 \mathrm{~ms}$. In [20], delay measurements in different broadband media delivery scenarios were performed. From those measurements, a worst-case analysis was made for each of the factors contributing to the delays, focusing on the video component of the multimedia contents. Those results are summarized in Table 1. It shows that encoding, transmission, and buffering are the main sources of video delays. Interestingly, it points out that differences in end-to-end video delays between receivers in an IPTV scenario can be larger than $6 \mathrm{~s}$ (with delays ranging between $250 \mathrm{~ms}$ and $6500 \mathrm{~ms}$ ).

\begin{tabular}{|c|c|c|}
\hline \multicolumn{2}{|r|}{ Delays } & Typical Delay Range (ms) \\
\hline \multirow[t]{5}{*}{ Source } & Video capture & $17-40$ \\
\hline & Video encoder & $50-2000$ \\
\hline & Encryption & $0-50$ \\
\hline & Error protection & $0-100$ \\
\hline & Output stream buffer & $0-500$ \\
\hline Uplink & Transmission & $10-300$ \\
\hline Processing & Transcoder & $0-2000$ \\
\hline Downlink & Transmission & $10-300$ \\
\hline \multirow[t]{5}{*}{ Home } & Input jitter buffer & $50-500$ \\
\hline & Error correction & $0-100$ \\
\hline & Decryption & $0-50$ \\
\hline & Decoder & $50-500$ \\
\hline & Display buffer & $0-50$ \\
\hline & Total & $250-6500$ \\
\hline
\end{tabular}

In [12] and [14], it was shown that delay differences when delivering media content using broadcast technologies and web-based (broadband) technologies can accumulate up to $8 \mathrm{~s}$. In [15], it was also proven that web-based (broadband) TV solutions could introduce more than 1 minute (up to 72s) delays compared to "regular" TV broadcast technologies. In addition, significant delay differences between receivers when using exactly the same TV delivery technology, setup combination (i.e., subscription type/quality) and equipment in all of them were noticed in that study. However, no numbers were provided due to the lack of sufficient measurements (from multiple geographically distributed sites, using specific or different types of technologies and setups).

Notice that the above delay magnitudes can vary (specially being reduced) whenever network operators will adopt nextgeneration technologies and infrastructures.

Another important factor that has a significant influence on delay and its variability is the existence of a precise, high resolution and reliable local timing mechanism. It is essential to have a coherent and precise notion of time in all the involved components along the end-to-end media delivery chain, as well as in the media delivery protocols in use [21]. For example, the importance of having an accurate source (reference) of time in all the involved consumption devices is emphasized in [22] and [15]. In [22], the effect of playout rate imperfections, due to clock skews/drifts, on delays and on delay differences is analyzed. In presence of clock skews/drifts, the delay differences for each individual media stream can originate buffer underflow/overflow (thus negatively affecting to intrastream sync). In addition, delay differences between media streams and between receivers can originate asynchrony situations (thus negatively affecting to inter-stream sync and IDES or IDMS, respectively). These delay differences can accumulate over time and need to be corrected, or, ideally, avoided. Examples of technologies for clock sync are NTP (Network time Protocol), PTP (Precise Time Protocol) and GPS (Global Positioning System). Nevertheless, the use of a clock sync technology is not enough. It needs to be properly set up and it also depends on its accuracy and support in specific devices. For example, in [15], it was found that GPS timing on Android devices was inaccurate, with fluctuations of up to $1 \mathrm{~s}$.

The aforementioned measurements may vary between media types and formats, technologies, regions and countries. These results show that relative differences occur and can degrade the perceived QoE in distributed TV media services.

In addition to these studies to determine the magnitudes of delays and delay differences, other previous studies have investigated the thresholds of delay variability ranges that are acceptable to the human perception, for different types of media sync solutions and use cases. In [23], different studies focused on determining the acceptable thresholds for jitter in audio and video streaming services (i.e., intra-stream sync), and for lipsync (i.e., audio and video sync, which is the most typical use case of inter-stream sync) are reviewed. These studies have revealed that jitter values of few ms (or even lower) can already be annoying in audio streaming services. Depending on the study and on the specific scenario, lip-sync asynchronies between a few tens of ms and a bit lower than $200 \mathrm{~ms}$ can be perceptible or annoying. Regarding inter-stream sync, the study in [24] provides a set of allowable asynchrony values between different types of media streams that may be tolerable to human perception.

Regarding IDES and IDMS, a large number of use cases can be found in [11], which are qualitatively ranked according to their sync requirements. The sync levels are: very high $(10 \mu$ s to $10 \mathrm{~ms}$ ); high (10 to $100 \mathrm{~ms})$; medium (100 to $500 \mathrm{~ms})$; and low (500 to $2000 \mathrm{~ms}$ ). For instance, networked stereo loud speakers require very high level sync; multi-party multimedia conferencing demands high level sync; second screen sync needs medium level sync; and finally, Social TV requires low level sync. Some related studies have been conducted, such as the ones in [20], [7], [13] and [25]. According to [20], the allowable asynchrony values for IDMS in interactive services may vary between 15 and $500 \mathrm{~ms}$, depending on the type of service. In some cases, differences around 100ms may already have an annoying effect on such interaction. Likewise, it is commonly accepted that the maximum end-to-end one-way delay when talking remotely should not exceed $150 \mathrm{~ms}$. Below this value, a user cannot perceive the delay in communication and, therefore cannot detect differences on sync of shared video content [7].

Moreover, controlled experimental setups have analyzed the effect of the existence of different asynchrony levels on the QoE in Social TV scenarios ([13] and [16]), in which remote users interacted via voice and text chat. In such testing, various synchronization conditions, with different asynchrony levels ranging from 0 to $4 \mathrm{~s}$ (in steps of $500 \mathrm{~ms}$ ), were forced and presented to participants in a randomized order, by enabling one of the two interaction channels (voice and text) in each test. It was concluded that, on the one hand, asynchrony values up to 1s might not be perceptible by users while communicating using audio conferencing services. On the other hand, playout time differences above $2 \mathrm{~s}$ really became annoying for most of 
them (i.e., both voice and text chatters). Concretely, when using voice chat, users noticed synchronization differences sooner, were more annoyed and felt more together than when using text chat. However, users with high text chat activity noticed sync differences similar to those using voice chat. Similar results were obtained in [16] by recreating a shared football watching experience. More recently, in [25], the influence of asynchrony values when consuming multimedia content together while being geographically distributed was also investigated. A subjective quality assessment using crowdsourcing was conducted in order to determine the allowable asynchrony thresholds in these scenarios. The results showed a significant decrease in the overall QoE when the value of the asynchrony level reached $750 \mathrm{~ms}$. At the same time, it was shown that an asynchrony level of $400 \mathrm{~ms}$ did not have an impact on the QoE compared to the synchronized reference case. Several allowable thresholds are also given in [26] for IDES use cases, such as $\pm 10 \mu$ s for tightly coupled audio; $2 \mathrm{~ms}$ for real-time audio; $15 \mathrm{~ms}$ for audio leading and $45 \mathrm{~ms}$ for audio lagging in lip-sync; and $\pm 80 \mathrm{~ms}$ for video animation.

Despite their relevance, the above results are highly dependent on several factors, such as the genre of the video content, the number of users, their activity and profiles (age, sex, relationships among them-family, friends, partners, etc....), the communication channel in use, etc. Consequently, no statistically absolute user tolerance limits may be derived from those experiments.

From the analyzed works, it can be concluded that the magnitudes of delay differences in current delivery technologies and scenarios are much larger than the tolerable asynchrony thresholds for the different types of media sync in different use cases. This fact motivates the need for adaptive hybrid sync solutions, to compensate for these differences.

\section{STUDY ABOUT THE SPANISH CONSUMERS' PERSPECTIVE ABOUT HYBRID TV MEDIA SERVICES}

This section presents the research study conducted to determine the Spanish consumers' habits, demands, opinions, preferences and expectations regarding hybrid TV media services. The selected use cases, the employed methodology, the sample, general data and a summary of the obtained results are presented in the different sub-sections.

\section{A. Selected Use Cases}

In order to conduct the study, 4 use cases in which different types of media sync are needed (inter-stream, IDES and/or IDMS), have been selected. They are the most representative use cases related with hybrid TV media services from the list of 19 use cases identified in [11], in which some kinds of media sync solutions are needed. Their selection is also based on their commercial potential, as discussed with interested agents (operators, content producers/providers), and on the fact that their service offering can take advantage of a seamless coordination between broadcast (DVB) and broadband (IP) technologies. Therefore, they are use cases with significant chances of successful deployment in Spain (and in other European and international countries driving the adoption of hybrid TV media services). Indeed, their relevance and popularity were corroborated in the study.
The selected use cases to ask consumers about are the following (corresponding with the examples $a$ to $d$ presented in Section II-A):

a) Case 1 - Online Sports Commentator: a consumer watching a sports match on $\mathrm{TV}$, while using a companion device to listen to an alternative commentator from an online radio channel.

b) Case 2 - Intelligence Quotient (IQ) Test TV program: a consumer watching a TV program about IQ Test on TV, while using an app on a companion device presenting the same questions as in the TV program. This allows the consumer virtually and remotely participating in the program.

c) Case 3 - Multi-view camera in Formula 1/Moto GP (Grand Prix): a consumer watching a Formula 1 or Moto GP race on $\mathrm{TV}$, having the possibility of dynamically selecting and switching between several camera views, either on the same device or on different devices.

d) Case 4 - Social TV, World Cup Final: a consumer watching the World Cup final on TV, while socially interacting and discussing about the match with remote friends.

\section{B. Methodology}

\section{Design of the Study}

A correlational (or descriptive) and cross-sectional study design [27] was used to conduct this research. The information from participants was obtained without manipulating the environment, by collecting evidence from the real world. Furthermore, the study involved just a one-time interaction with the participants, by collecting the targeted information via an anonymous online questionnaire.

In Section IV.C, general data about the study are provided.

\section{Employed Tools}

The participants may not have previously experienced with the use case before, because, up to the date the survey took place, they were not exactly implemented using hybrid technologies in Spain (neither in Europe) yet. As far as authors know, very few similar services to the four selected use cases can be found (described later). Moreover, the existing ones include a weak support and poor integration in multi-screen scenarios. Similar services to use case 2 were preliminarily introduced, e.g. in The Netherlands and Spain. They mainly used proprietary and not highly accurate solutions to achieve sync of the media playout on companion devices, such as fingerprinting or watermarking. A service similar to the use case 3 was experimentally offered, before the period of time when the study took place, by Mediaset, Radio Televisión Española (RTVE) and TV3 (Spanish broadcasters). It uniquely used broadcast or broadband technologies (without combining them). Services similar to use case 4 had not been offered yet in Spain, although some related research projects preliminarily experienced with them (discussed later) in other countries.

Accordingly, the designed questionnaire included an exhaustive explanation of each use case, with some illustrations, to help participants to identify them in a more friendly way. Up to 55 (open and closed) questions, including multiple-choice questions were included in the questionnaire. 
Depending on the provided answers, the flow and the number of the presented questions could vary for each participant. That is the reason why some questions were not answered by all of them. Nevertheless, all the questions were answered by, at least, $30 \%$ of participants. The questionnaire was designed to be completed in less than 30 minutes.

The survey was publicly available through the on-line SurveyMonkey ${ }^{\circledR}$ web-based platform ${ }^{11}$. It allows creating, storing and disseminating from simple polls to in-depth research studies or surveys. By using that platform, more responses could be collected in less time compared to the use of alternative methods (e.g. individual interviews). It allowed checking the progress regarding users' participation and the collected responses at any time (e.g., by inspecting individual responses, summaries, grouped statistics, etc.). It also allowed inviting participants to complete the questionnaire via different channels (web/blog, e-mail and Social Media tools, such as WhatsApp, LinkedIn, Facebook or Twitter). At the time of writing this paper, the questionnaire was still available at http://svy.mk/2f9gp4k. The participants could fill in it individually and anonymously. Neither personal data nor credentials about them were collected.

\section{Sample and participants' data}

In order to get representative, generalizable and conclusive results in the study, a convenience sample of the population was required [28]. In particular, a random sample of 1015 Spanish participants was considered. It guaranteed an error lower than $3.1 \%$ for a $95 \%$ Confidence Interval. The participation occurred from 1st June 2015 to 31st December 2015 (7 months).

All the participants were older than 18 , and $44.83 \%$ of them were men. Figure 3 represents their age, occupation, study level and profile distributions.

\section{Results}

This Section provides a summary of the most relevant results collected in the study. It includes a sub-section for general results and one sub-section for particular results for each use case. Although the results have been collected from Spanish participants, their main conclusions can be extrapolated to other countries driving the adoption of hybrid TV media services. Indeed, the obtained data and statistics have been corroborated to be consistent with the feelings and data statistics of members of the HbbTV organization from other countries, during the last HbbTV Symposium (in October 2016).

The questionnaire was publicly available (i.e., no geographical restrictions on its access were applied), and it was published and shared in different Social Media and communication channels during the period in which the study took place. Consequently, more than 200 additional responses from foreign participants (all around the world) were collected. Those additional responses were filtered out and not considered in the results presented in this paper. Close to 150 of those additional responses were from participants from other European countries with hybrid TV media services (Germany,

\footnotetext{
${ }^{11}$ https://www.surveymonkey.com/
}

The Netherlands and France). Just out of curiosity, we also analyzed them and, although we recognize that they do not constitute a representative sample, they were very similar (in percentages) to the ones presented in this paper for Spanish consumers.

\section{General Results}

$90.1 \%$ of participants declared to watch some variant of TV (e.g., linear broadcast, IPTV or web-based). 23.5\% declared they did not have a Smart TV, but they would like to buy one soon; $30.4 \%$ neither had nor intended to buy one yet; $19.4 \%$ had one and thought that its Internet connectivity features provided an added value when watching TV; and $10.9 \%$ had one, but did not use such features.

Regarding the use of companion devices while watching TV, $25.9 \%$ of participants declared they used them while watching $\mathrm{TV} ; 32.2 \%$ did not, but thought they could provide enriched TV watching experiences; and $25.1 \%$ were not interested at all in using them. The latter found them to be distracting and intrusive to their TV watching experience.

Smartphones were the most popular companion devices used by second-screeners $(78.3 \%)$, followed by tablets $(55.4 \%)$, laptops (41.9\%) and PCs (0.8\%). Android (64.7\%) was the most commonly used Operating System, followed by iOS (41.1\%), Windows $(21.78 \%)$ and Linux $(0.4 \%)^{12}$.

Regarding the possibility of being automatically provided with extra (related) content while watching TV, only $11.6 \%$ of participants were not interested, because they just wanted to sit down and passively watch TV (i.e., leaning back experience); $15.1 \%$ were interested, but preferred to search it by themselves; $26.4 \%$ were interested, but only for specific TV genres; and $31.3 \%$ were really interested $(36.5 \%$ of them preferred to watch it on the same main screen, $22.9 \%$ on a secondary screen, and the rest did not mind whatever screen the extra content was presented on). All the participants interested in extra-related content were asked about the TV genres they would like to be provided with it. The results are shown in Table 2, including the distribution of responses regarding the participant's sex.

Regarding demands about the extra-related content, participants mainly declared they wanted it to be continuously updated (60.5\%); reliable (49.6\%); synchronized with the other contents being consumed (45\%); and to be able to filter the extra content types (24\%) and sources (20.28\%). Just 6.2\% did not demand anything regarding this.

\section{Results for each Use Case}

A high percentage of participants did show interest in each one of the use cases (only 9.3, 8.3, 20.6 and 21.5\% did not, respectively). The main results for each use case are presented in the next sub-sections. In particular, participants were asked about their similar previous experiences and interest in the use case (Table 3), their expectations (Table 4), their sensations in case of previous experience with them (Table 5), the identified benefits (Table 6) and limitations (Table 7), among other relevant issues.

\footnotetext{
12 The sum of percentages exceeds the amount of $100 \%$ because it was a multiple-choice question (as in many other questions).
} 

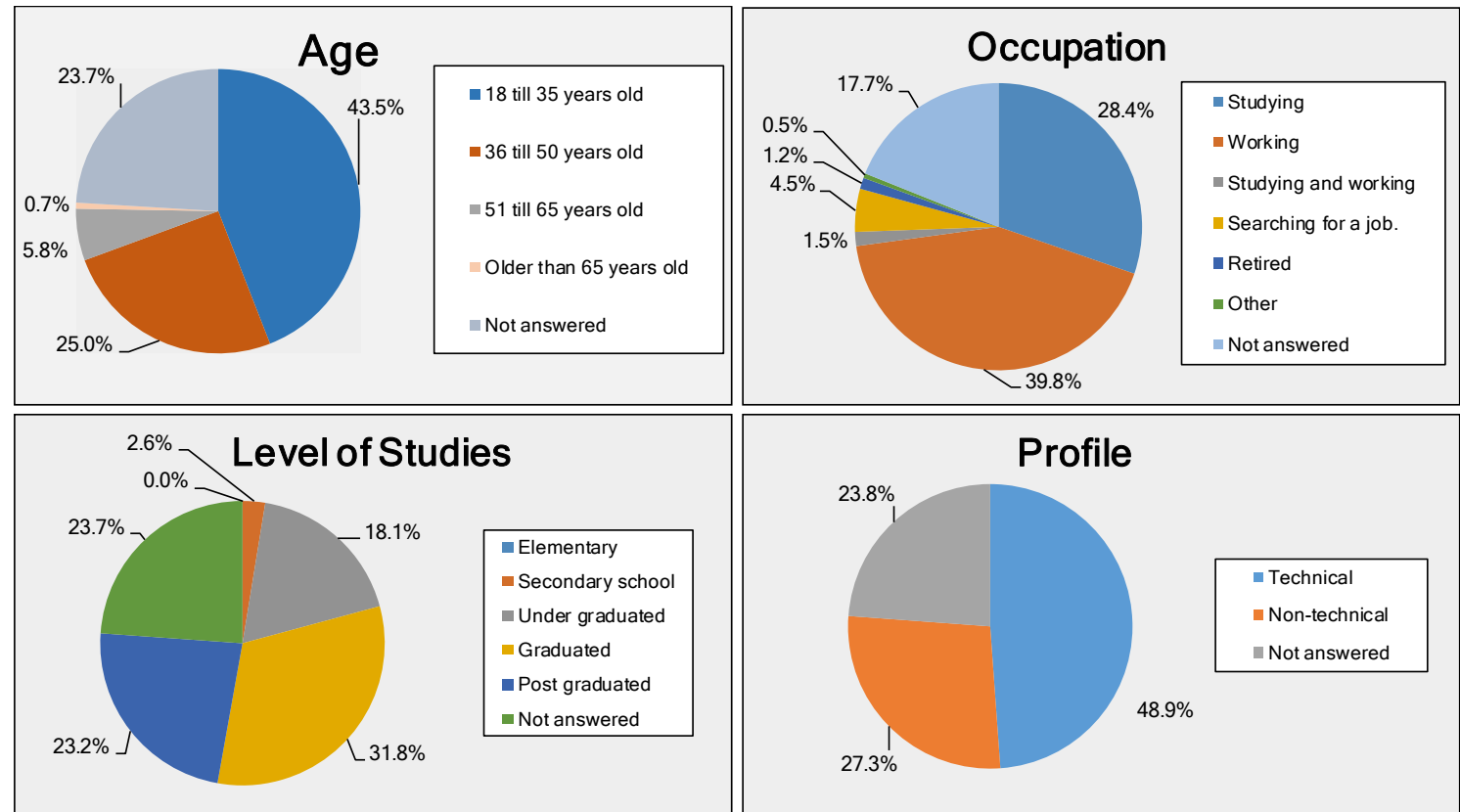

Fig. 3. General Data about Participants.

TABLE 2. TV GENRES WITH DEMAND OF EXTRA (RELATED) CONTENT

\begin{tabular}{||c|rc||}
\hline \hline TV Genre & $\begin{array}{r}\text { Percentage of participants (\%) demanding } \\
\text { extra (related) content } \\
\text { (\% male/female of the overall value) }\end{array}$ \\
\hline \hline Films & 58.20 & $(47.77 / 52.23)$ \\
\hline Series & 52.00 & $(45.08 / 54.92)$ \\
\hline News & 43.25 & $(54.27 / 45.73)$ \\
\hline Non-Fiction & 36.30 & $(53.47 / 46.53)$ \\
\hline Sports & 34.80 & $(76.84 / 23.16)$ \\
\hline Music & 34.40 & $(50.06 / 49.94)$ \\
\hline Games & 15.80 & $(64.94 / 35.06)$ \\
\hline Reality shows & 13.60 & $(48.53 / 51.47)$ \\
\hline Porn & 2.60 & $(84.62 / 15.38)$ \\
\hline \hline
\end{tabular}

a) Case 1: Online Sports Commentator

$90.7 \%$ of participants declared having interest in this use case, of whom $50.27 \%$ were male, but just $24.7 \%$ affirmed having participated in similar experiences before (of whom $78.53 \%$ were male) as presented in Table 3. Such experiences mainly consisted of watching events (e.g., sports or award ceremonies) on TV while listening to alternative (FM or online) radio channels commenting about the event.

The main participants' expectations (Table 4) were: they wanted the on-line audio to be in sync with the content on the main screen $(78.1 \%)$; to be able to watch TV events on foreign channels, but listening to a native commentator (49.6\%); and to be provided with complementary information (41.4\%).

Regarding their perceived sensations (Table 5), 37.5\% experienced negative or very negative ones, while $35.7 \%$ experienced positive or very positive ones. On the one hand, the main reason for the negative ones was the lack of sync (i.e., temporal alignment) between the TV content and the radio (audio) content. On the other hand, the main reason for the positive sensations was the possibility of choosing a radio channel with their preferred commentator, or in their native language (for foreign events or consumers).
TABLE 3. SIMILAR EXPERIENCES AND INTEREST IN USE CASES

\begin{tabular}{||c||c||c|}
\hline \multicolumn{1}{|c|}{ Use case } & \multicolumn{2}{|c|}{$\begin{array}{c}\text { Percentage of participants (\%) } \\
\text { (\% Male/Female of the overall value) }\end{array}$} \\
\cline { 2 - 3 } & $\begin{array}{c}\text { Participants with similar } \\
\text { previous experiences }\end{array}$ & $\begin{array}{c}\text { Participants interested } \\
\text { in this use case }\end{array}$ \\
\hline \hline $\begin{array}{c}\text { 1. Online Sports } \\
\text { Commentator }\end{array}$ & $\begin{array}{c}24.7 \\
(78.53 / 21.47)\end{array}$ & $\begin{array}{c}90.7 \\
(50.27 / 49.73)\end{array}$ \\
\hline \hline $\begin{array}{c}\text { 2. IQ Test TV } \\
\text { program }\end{array}$ & $\begin{array}{c}8.5 \\
(60.27 / 39.73)\end{array}$ & $\begin{array}{c}91.7 \\
(61.04 / 38.96)\end{array}$ \\
\hline \hline $\begin{array}{c}\text { 3. Multi-view } \\
\text { camera in F1/Moto } \\
\text { GP }\end{array}$ & 7.1 & 79.4 \\
$(71.88 / 28.13)$ & 78.5 \\
\hline $\begin{array}{c}\text { 4. Social TV, World } \\
\text { Cup Final }\end{array}$ & $\begin{array}{c}21.3 \\
(56.22 / 43.78)\end{array}$ & $(61.13 / 38.87)$ \\
\hline
\end{tabular}

The main identified benefits (Table 6), in percentage, for this use case were: $68.1 \%$ declared they were provided with a more enjoyable and personalized experience; $52.8 \%$ stated those kinds of applications could contribute to a better social integration; and $40.6 \%$ considered that this use case could provide extra knowledge/information about the broadcast TV event. Only $1.6 \%$ did not see any benefit. On the other hand, most of participants identified technical drawbacks (Table 7) as the main limitations in this use case (e.g., the lack of sync by $78.1 \%)$.

Participants were also asked for their opinion about whether a technology to seamlessly substitute the default audio content by an alternative one (e.g., provided by an FM or on-line radio station, and consumed either on the main or on a companion device), while watching TV, was relevant and useful. Figure 4 summarizes their responses. $4.8 \%$ believed such functionality was not interesting at all; $25.5 \%$ declared that it was, but solutions to achieve it already existed (with an acceptable performance); and $69.7 \%$ thought that a proper technology for seamless audio substitution was interesting, and that (better) solutions were still needed. 


\section{b) Case 2: Intelligence Quotient (IQ) Test TV program.}

$91.7 \%$ of participants (of whom $61.04 \%$ were male) declared interest in this use case, but only $8.5 \%$ (of whom 60.27 were male) affirmed having participated in similar experiences before (Table 3). Those experiences mainly consisted of watching 'Atrapa un millón'13 and 'Letris'14 programs. Both programs were broadcast by national TV channels, and allowed users participating in the TV program by using a second screen app.

Regarding their expectations (Table 4), participants mainly wanted the questions on TV to be in sync with the ones on the companion device $(60.8 \%)$ and to be able to fairly compete with other participants $(52.4 \%)$.

$7.4 \%$ of participants declared having experienced negative or very negative sensations in this kind of use cases, while $62.9 \%$ declared having experienced positive or very positive ones (Table 5). The main reasons for the negative ones were the lack of sync and the bad performance (high delays, non-stability...) of the provided apps. Participants with positive sensations declared that it was fun to compete against other participants and to feel like virtual participants (despite the lack of sync noticed at some instants).

Regarding benefits provided by this use case (Table 6), $74.3 \%$ of participants declared they had a more enjoyable and personalized experience; $49.6 \%$ stated that they could compete with other remote participants; and $50.4 \%$ liked to compare their IQs to the other participants' (including the ones at the TV show). Only $2.2 \%$ did not see any benefit. Technical problems were also identified as the main limitations (Table 7) in this case (e.g., lack of sync for $78.1 \%$ ).

Participants were also asked whether this use case provided them a satisfactory feeling of engagement. $83.3 \%$ agreed (partially, $45 \%$, or completely, $38 \%$ ), while only $4.7 \%$ disagreed (partially, $2 \%$, or completely, 2.7 .

Most of the participants (more than $90 \%$ ) also thought that a proper technology for virtually and remotely participating in TV shows/programs and competing with other contestants from home, by using second screen apps, was interesting (Figure 4).

\section{c) Case 3: Multi-view camera in Formula 1/Moto GP.}

In this case, $79.4 \%$ of participants (of whom $62 \%$ were male) declared having interest in this use case, but just $7.1 \%$ (of whom $71.88 \%$ were male) declared having participated in similar experiences before (Table 3). The reported experiences consisted of watching F1 or Moto GP races, awards ceremonies, music festivals or playing games, with multi-view or multicamera functionalities. Nevertheless, at that time, such services were offered using just one delivery technology (broadcast or broadband, depending on the operator and service) and for onescreen scenario (e.g., a Smart TV). No multi-screen capabilities were offered in Spain for those services.

Regarding their expectations about multi-view TV services (Table 4), participants mainly wanted to have a more engaging and personalized experience (77.5\%), and many of them
(55.5\%) expected the multi-view contents to be presented in sync on all the involved consumption devices.

In this use case, just $1.5 \%$ of the participants experienced negative or very negative sensations, while $70.2 \%$ experienced positive or very positive ones (Table 5). The main reasons for the negative ones were the lack of sync, insufficient bandwidth availability, long zapping delays when switching between cameras/views and distraction (i.e., loss of content and, therefore, of information) when enabling the multi-view functionality. Participants with positive sensations mainly declared that it was fun to be able to choose by themselves the view from which to experience the event, and that it provided an engaging, more enjoyable and personalized experience. The high percentage of positive sensations could be explained because no hybrid TV media services and no multi-screen capabilities were really provided. Although it was transparent for users, just broadcast or broadband delivery was used, without a coordination between the two technologies. Accordingly, in the mentioned similar experiences, the associated lack of sync between technologies and between consumption devices do not apply, as they were not really experienced by users. Therefore, these effects were not a cause for the experienced negative sensations.

As benefits (Table 6), 76.2\% declared this use case provided a more enjoyable and personalized experience; $69.7 \%$ stated it could provide other interesting points of view; while $41.4 \%$ stated that it was useful to get extra knowledge about the event. Only $0.6 \%$ did not see any benefit. As limitations (Table 7), most users also identified technical problems (e.g., lack of sync for $64.3 \%$ ).

Most participants also thought that a proper technology to provide personalized and enriched multi-view TV experiences was interesting (Figure 4). Just only $2.6 \%$ of participants disagreed with this.

\section{d) Case 4: Social TV, World Cup Final.}

In this case, $78.5 \%$ of participants (of whom $61.13 \%$ were male) declared having interest in this use case (Table 3), stating that they mainly wanted to watch TV alone or with other physically co-located consumers.

Participants interested in these kinds of experiences were also asked about what TV genres they would like to share experiences with remote consumers. The results are in Table 8, including the distribution of responses regarding the participant's sex.

$21.3 \%$ (of whom $56.22 \%$ were male) declared having participated in similar experiences before (Table 3). Such experiences mainly consisted of commenting on Twitter, WhatsApp, Skype, or official TV programs apps, with friends or relatives while watching TV series, reality shows or sports events.

\footnotetext{
${ }^{14}$ http://www.rtve.es/television/letris/ (last access: January 2017)
}

${ }^{13} \mathrm{http}: / /$ www.antena3.com/programas/millon// (last access: January 2017) It is similar to 'Who wants to be a millionaire' program. 
TABLE 4. PARTICIPANTS' EXPECTATIONS IN EACH USE CASE

\begin{tabular}{|c|c|c|}
\hline Use Case & Expectations & Answers (\%) \\
\hline \multirow{5}{*}{$\begin{array}{l}\text { 1. Online Sports } \\
\text { Commentator }\end{array}$} & The on-line audio is in sync with the content on the main screen. & 78.1 \\
\hline & To be able to watch sports events in foreign channels, but listening to a native (Spanish) commentator. & 49.6 \\
\hline & To be provided with complementary information. & 41.4 \\
\hline & To be able to select and switch between different languages. & 24.0 \\
\hline & I do not expect anything. & 6.2 \\
\hline \multirow{5}{*}{$\begin{array}{l}\text { 2. IQ Test TV } \\
\text { program. }\end{array}$} & Questions on the companion device (or screen) are in sync with the ones on TV (main screen). & 60.8 \\
\hline & To be able to (fairly) compete with TV contestants and/or other remote participants. & 52.4 \\
\hline & To compare my IQ with the ones of other participants. & 49.8 \\
\hline & Increased engagement. & 47.4 \\
\hline & I do not expect anything. & 2.2 \\
\hline \multirow{6}{*}{$\begin{array}{l}\text { 3. Multi-view } \\
\text { camera in } \\
\text { F1/Moto GP }\end{array}$} & To have a more engaging and personalized experience. & 77.5 \\
\hline & The contents being consumed should be synchronized on all the involved devices. & 55.5 \\
\hline & Access to information from other angles or points of view. & 1.2 \\
\hline & More comfortable information access. & 0.2 \\
\hline & To be able to watch whatever I want, without anybody deciding for me. & 1.0 \\
\hline & I do not expect anything. & 3.1 \\
\hline \multirow{5}{*}{$\begin{array}{l}\text { 4. Social TV, } \\
\text { World Cup Final. }\end{array}$} & To obtain the same information at exactly the same time compared to the other involved consumers (accurate sync). & 46.5 \\
\hline & To obtain the same information at slightly different instants compared to the other involved consumers. & 26.5 \\
\hline & To be provided with ad-hoc text, audio and/or video chat tools integrated with the shared experience. & 26.5 \\
\hline & $\begin{array}{l}\text { To be able to use my own text, audio and/or video chat tools (e.g. WhatsApp, Skype, Twitter, etc.), even with a poor } \\
\text { integration with the shared experience. }\end{array}$ & 27.9 \\
\hline & I do not expect anything. & 8.8 \\
\hline
\end{tabular}

TABLE 5. CONSUMERS' SENSATIONS IN EACH USE CASE

\begin{tabular}{||c|c|c|c|c||}
\hline \hline Sensation & 1. Online Sports Commentator (\%) & 2. IQ Test TV program (\%) & 3. Multi-view camera (\%) & 4. Social TV (\%) \\
\hline \hline Very negative & 2.1 & 0.0 & 0.0 & 0.0 \\
\hline Negative & 35.4 & 7.4 & 2.5 & 6.5 \\
\hline Neutral & 26.6 & 29.6 & 28.4 & 26.3 \\
\hline Positive & 31.2 & 48.1 & 20.0 & 20.9 \\
\hline Very positive & 4.6 & 14.8 & 20.9 \\
\hline
\end{tabular}

TABLE 6. IDENTIFIED BENEFITS FOR EACH USE CASE

\begin{tabular}{|c|c|c|}
\hline Use Case & Benefits & Answers (\%) \\
\hline \multirow{6}{*}{$\begin{array}{l}\text { 1. Online Sports } \\
\text { Commentator }\end{array}$} & A more enjoyable and personalized experience. & 68.1 \\
\hline & It can contribute to social integration. & 52.8 \\
\hline & To get extra knowledge about the event. & 40.6 \\
\hline & It can help to learn other languages. & 0.7 \\
\hline & To be able to select a better commentator. & 0.5 \\
\hline & I do not see any benefit. & 1.6 \\
\hline \multirow{5}{*}{$\begin{array}{l}\text { 2. IQ Test TV } \\
\text { program }\end{array}$} & A more enjoyable and personalized experience (it is fun to test the own IQ) & 74.3 \\
\hline & I can compete against other participants who are far away from me. & 49.6 \\
\hline & I can compare my IQ to the IQs of other participants. & 50.4 \\
\hline & Increased engagement. & 0.2 \\
\hline & I do not see any benefit. & 2.2 \\
\hline \multirow{5}{*}{$\begin{array}{l}\text { 3. Multi-view } \\
\text { camera in F1/Moto } \\
\text { GP }\end{array}$} & A more enjoyable and personalized experience (e.g., I can continuously follow the race of my favorite driver). & 76.2 \\
\hline & It provides other interesting points of view of the event (e.g., paddock, pit lane, backstage, etc.). & 69.7 \\
\hline & It is interesting and useful to get extra knowledge about the TV content. & 41.1 \\
\hline & To feel as a producer. & 0.4 \\
\hline & I do not see any benefit. & 0.6 \\
\hline \multirow{5}{*}{$\begin{array}{l}\text { 4. Social TV, } \\
\text { World Cup Final }\end{array}$} & It provides a feeling of 'togetherness', even though the involved users are geographically distributed. & 69.4 \\
\hline & It is interesting to know what the other users think about the event. & 43.8 \\
\hline & Having a shared experience is the reason why I watch specific TV events (e.g., sports events, TV shows, etc.). & 31.2 \\
\hline & Increased engagement (higher immersion). & 25.0 \\
\hline & I do not see any benefit. & 4.3 \\
\hline
\end{tabular}


TABLE 7. IDENTIFIED LIMITATIONS FOR EACH USE CASE

\begin{tabular}{|c|c|c|}
\hline Use Case & Limitations & Answers (\%) \\
\hline \multirow{6}{*}{$\begin{array}{l}\text { 1. Online Sports } \\
\text { Commentator }\end{array}$} & Technical drawbacks, such as lack of sync. & $\overline{78.1}$ \\
\hline & Other technical drawbacks (e.g., insufficient bandwidth availability or low-speed connection). & 62.5 \\
\hline & Confusion when original commentators are on screen in specific moments, but you do not listen to them. & 18.4 \\
\hline & It could be disturbing and annoying. & 0.7 \\
\hline & Lack of technological education. & 0.5 \\
\hline & I do not see any limitation. & 5.2 \\
\hline \multirow{3}{*}{$\begin{array}{l}\text { 2. IQ Test TV } \\
\text { program }\end{array}$} & Technical drawbacks, such as lack of sync. & 60.8 \\
\hline & Other technical drawbacks (e.g., insufficient bandwidth availability or low-speed connection). & 61.0 \\
\hline & I do not see any limitation. & 18.0 \\
\hline \multirow{6}{*}{$\begin{array}{l}\text { 3. Multi-view } \\
\text { camera in F1/Moto } \\
\text { GP }\end{array}$} & Technical drawbacks, e.g., when the selected views are not presented in sync (i.e., lack of sync). & 64.3 \\
\hline & Technical drawbacks (e.g., insufficient bandwidth availability or low-speed connection). & 64.7 \\
\hline & Distraction. & 1.2 \\
\hline & Cost of companion devices. & 0.6 \\
\hline & Technology is not ready yet. & 0.4 \\
\hline & I do not see any limitation. & 10.6 \\
\hline \multirow{6}{*}{$\begin{array}{l}\text { 4. Social TV, World } \\
\text { Cup Final }\end{array}$} & $\begin{array}{l}\text { Technical drawbacks, when not all the participants perceive the same events/actions at the same time (i.e., lack } \\
\text { of sync). }\end{array}$ & 55.9 \\
\hline & Technical drawbacks, when a participant cannot access the same content as the other participants. & 48.0 \\
\hline & Other technical drawbacks (e.g., insufficient bandwidth availability or low-speed connection). & 53.7 \\
\hline & The participants may not have the same chat tool at their disposal. & 25.1 \\
\hline & Distraction. & 27.2 \\
\hline & I do not see any limitation. & 6.9 \\
\hline
\end{tabular}

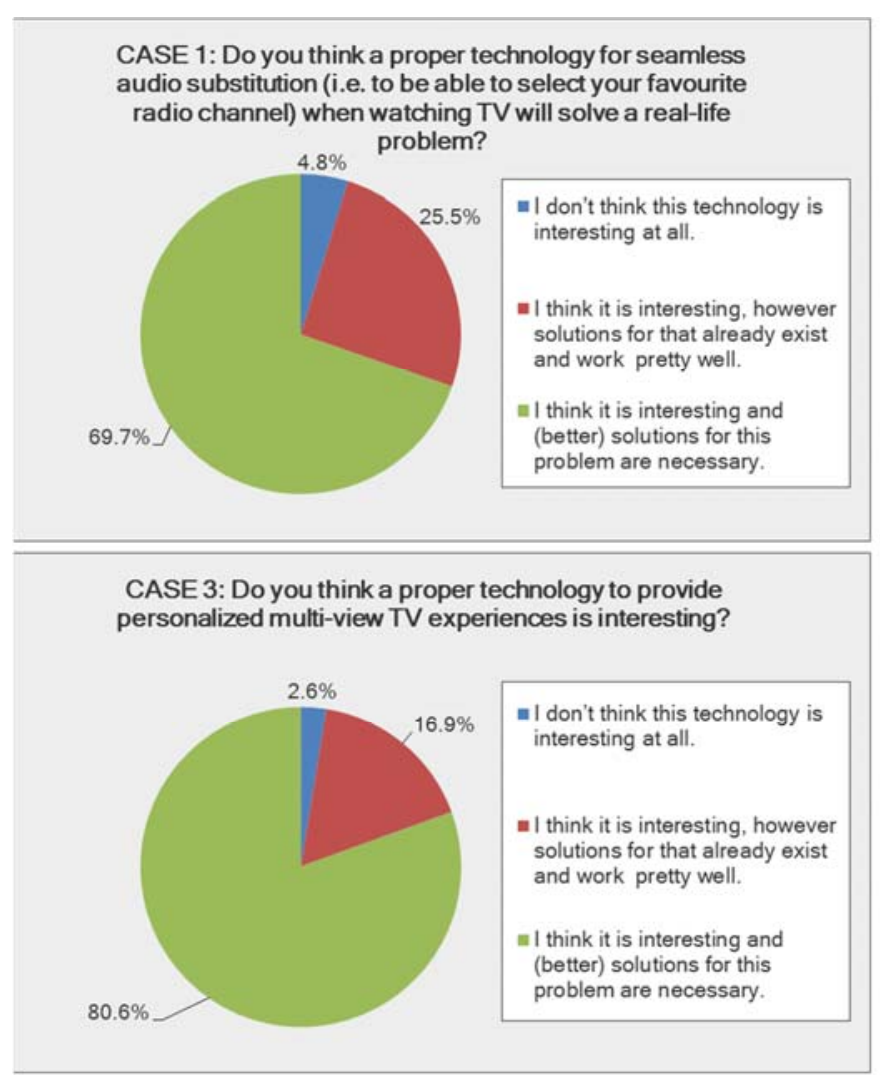

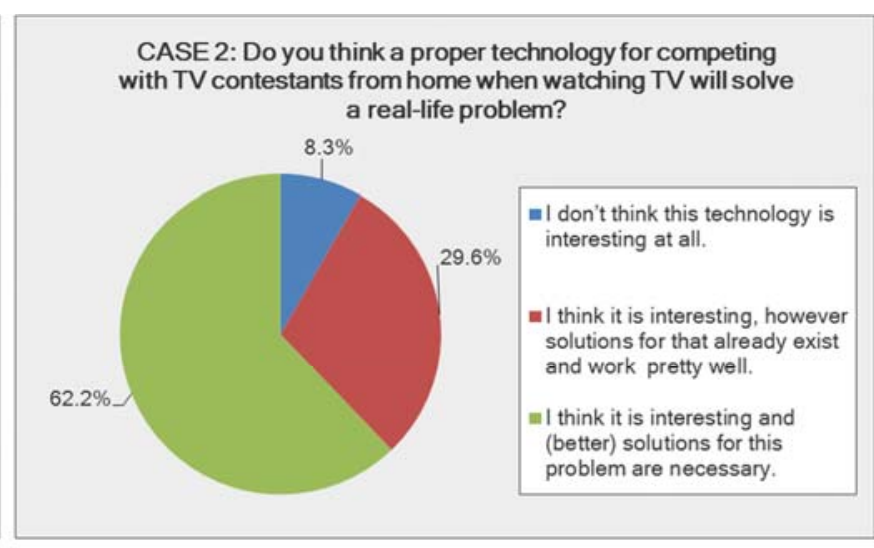

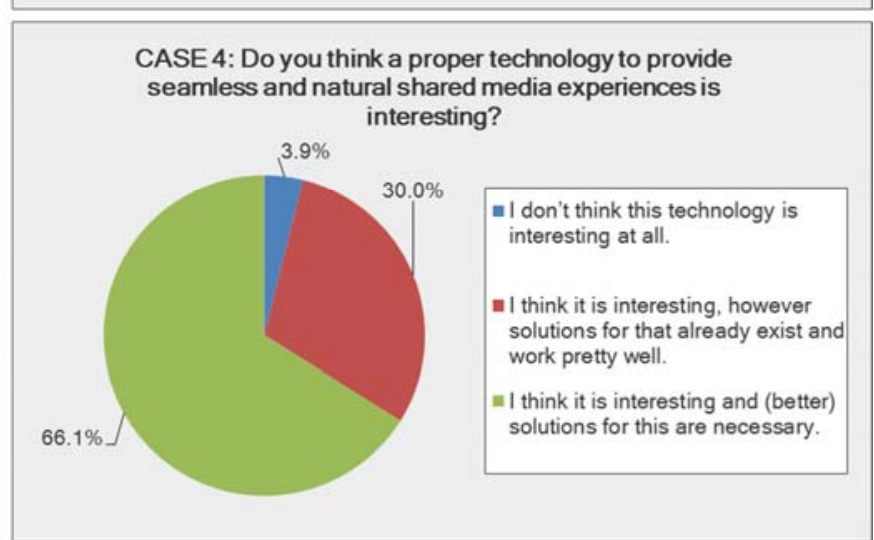

Fig. 4. Participants' opinion about the current technology support for each use case. 
Regarding the participants' expectations (Table 4) from Social TV as a means to provide shared media experiences, they mainly wanted to perceive the information/events related to the media contents (almost) at the same time than (i.e., in sync with) the other involved consumers.

In this case, only $6.5 \%$ experienced negative or very negative sensations, while $81.4 \%$ experienced positive or very positive ones (Table 5). The main reasons for the negative ones were the lack of sync, distraction, and the lack of a 'real', natural and a comfortable interaction channel with text messages. Interacting with text messages makes a fluid communication difficult and can be distracting. Participants with positive sensations mainly declared that: i) it was fun to share the experience with remote consumers; ii) it provided a more enjoyable experience; and iii) they felt more engaged to the content being consumed (higher immersion).

TABLE 8. TV GENRES WITH DEMAND OF SHARED EXPERIENCES

\begin{tabular}{||c|rc||}
\hline TV genre & $\begin{array}{r}\text { Percentage of participants (\%) demanding } \\
\text { shared experiences } \\
\text { (\% male/female of the overall value) }\end{array}$ \\
\hline \hline Films & 42.10 & $(50.95 / 49.05)$ \\
\hline Series & 46.20 & $(47.62 / 52.38)$ \\
\hline News & 25.10 & $(70.76 / 29.24)$ \\
\hline Non-Fiction & 8.90 & $(72.02 / 27.98)$ \\
\hline Sports & 63.20 & $(69.19 / 30.81)$ \\
\hline Music & 24.50 & $(64.90 / 35.10)$ \\
\hline Games & 51.80 & $(62.36 / 37.64)$ \\
\hline Reality shows & 32.30 & $(49.16 / 50.84)$ \\
\hline
\end{tabular}

As benefits (Table 6), 69.4\% of participants declared this use case provided a more enjoyable and personalized experience, with a feeling of 'togetherness' with remote consumers with strong social bonds or with common interests; $43.8 \%$ stated that it was interesting to know about the thoughts of other consumers; $31.2 \%$ stated that sharing the experience was one of the reasons why they watch TV; while $25 \%$ considered the consequent engagement as a benefit. Only $4.36 \%$ did not see any benefit. As limitations (Table 7), technical problems were again identified as the most common drawbacks (e.g., lack of sync for $55.9 \%$ ).

Participants with previous experience in similar scenarios to this use case were also asked about three additional issues:

1) About the frequency of their interactions with remote consumers while watching TV. 7\% declared to interact once a year; $22.8 \%$ from one to ten times a year; $29.2 \%$ from one to three times a month; $21.1 \%$ from one to six times a week; $8.4 \%$ once a day; and $11.4 \%$ more than once a day.

2) About whether the communication delays and their variability between locations could prevent from satisfactorily enjoying shared TV experiences. $4.2 \%$ declared they had not perceived any delay differences in their previous experiences; $38.2 \%$ stated that, although delay differences were noticed, their magnitudes were not a serious barrier for satisfactorily watching TV with remote users; finally, $52.8 \%$ stated that delay differences prevented them from enjoyable shared experiences.

3) About what interaction modality they considered the most

${ }^{15}$ Authors expect this data will change soon since, three months after its launch, WhatsApp IP-phone tool overtook Skype tool. appropriate to increase the feeling of 'togetherness' with remote consumers. $54.0 \%$ answered text chat; $11.1 \%$ answered audio chat; and $34.8 \%$ answered video chat tools. WhatsApp (90.4\%), Facebook (40.4\%), Twitter (27.3\%) and Telegram (7\%) were the most commonly used text-based communication tools, while Skype (29.4\%) was the most used audio/video conferencing tool ${ }^{15}$. Other tools, such as SMS, Snapchat and Wechat, were also pointed out by a minority of participants (less than 1\%).

Finally, all participants were also asked about whether they considered interesting the availability of technologies capable of minimizing the magnitudes of delays, and of compensating their variability between remote consumers (i.e., of providing IDMS), to provide pleasant shared media experiences (Figure 4). $3.9 \%$ thought that such technologies were not interesting at all; $30 \%$ thought there were, however, solutions for that, which already existed and provided quite a satisfactory performance; and $66.1 \%$ thought they were interesting and that (better) technological solutions were still necessary.

\section{DISCUSSION}

In this Section, the results presented in the previous one are discussed, structuring them in different parts. Some of them are compared with the ones from related studies and some discussion is supported with actual facts and initiatives in this area.

\section{A. Hybrid Technologies and Smart TVs}

As mentioned before, the advent of hybrid media delivery technologies, combined with social media communications, has opened the door to a new wave of innovative and fascinating TV media services. Thus, consumers do not have to be restricted to single delivery technologies for TV media consumption, but they can concurrently consume media contents delivered via hybrid technologies. On the other hand, they do not have to passively consume TV contents in an isolated manner, but rather can interact with the hybrid TV media contents as well as with other remote users, thanks to the availability of bidirectional broadband communications.

The introduction of connected Smart TVs and companion devices, together with new interactive applications and services, are definitely impacting the way people watch TV. Therefore, more interactive, personalized and enjoyable experiences become available. It has also shifted the focus of the interactive TV industry to the introduction of interactive second screen apps. As a parallel trend, consumers are also commonly multi-tasking on the many available screen-based devices while watching TV.

Nevertheless, the proliferation of Smart TVs is still not widespread. At the date the study was conducted, a high percentage of Spanish consumers, 30.4\%, were not interested in Smart TVs yet. Only $30.3 \%$ had a Smart TV (quite a low percentage), but $23.5 \%$ declared intentions to buy one soon (both summing up to 53.8\%). These statistics can be compared 
with the ones obtained in 'The Smart TV Experience' study ${ }^{16}$, conducted in the UK, in 2015. According to it, more than half of participants in the UK did not own a Smart TV, compared to just over a third of participants in the US and just less than a fifth in China. In Table 9, the evolution of the Smart TV household penetration in the last two years in some European countries is provided, according to the study in [29].

TABLE 9. EVOLUTION OF THE SMART TV HOUSEHOLD PENETRATION IN EUROPE [28]

\begin{tabular}{||c|c|c||}
\hline \hline Country & $2015(\%)$ & $2016(\%)$ \\
\hline \hline Germany & 42 & 53 \\
\hline Spain & $\mathbf{3 8}$ & $\mathbf{4 8}$ \\
\hline Italy & 36 & 44 \\
\hline UK & 36 & 43 \\
\hline France & 35 & 40 \\
\hline
\end{tabular}

Consumers still have to be convinced that Smart TVs can provide them compelling media consumption experiences as standalone connected devices for their households. This is particularly relevant for older consumers, who are typically used to employing traditional systems (e.g. remote control and broadcast TV). They are not as predisposed as young consumers to test or subscribe to new services, unless the obtained benefits are clear. Many people are still unaware that these devices allow them watching whatever they want and whenever they want, without being restricted by broadcast TV schedules any more.

Additionally, for many consumers, Smart TVs are no longer the 'go to' connected media devices. They are progressively incorporating companion devices for (hybrid) media consumption. Accordingly, they still should be taught and motivated about the consumption of hybrid media contents on Smart TVs. More efforts on education and marketing campaigns, promoting the existence of hybrid related contents and the possibilities of their associated services (combined with a larger offer) should be provided to encourage consumers to acquire, and use, Smart TVs (alone or with companion devices).

B. Current initiatives to boost hybrid TV media services (in Spain)

In Spain, the popularity of Smart TVs is progressively increasing thanks to the recent landing of OTT (Over-The-Top) SVOD (Subscription Video on Demand) services, such as Netflix (October 2015). Such services are giving a boost to the pay-TV market, especially for contents related to sports (e.g., football championships, Olympics...), films and series. According to the last 2016 report $^{17}$ of the Comisión Nacional de los Mercados y la Competencia ${ }^{18}$ (CNMC), Yomvi (Movistar) has 928.000 clients (7.8\%); Netflix (Orange) has 216.000 customers $(1.8 \%$ of Spanish homes with Internet connection); Wuaki has 127.000 clients $(1,1 \%)$; and others, such as Filmin, Tucut and Nubeox, together exceed 90.000

$16 \mathrm{https}: / / \mathrm{www}$. strategyanalytics.com/access-services/devices/connectedhome/connected-home-ux/reports/report-detail/the-smart-tvexperience\#.VelcmRHBzGc, August 2015 (last access: January 2017)

${ }^{17}$ http://data.cnmc.es/datagraph/jsp/inf trim.jsp (last access: January 2017)

${ }_{18}$ Spain's Markets and Competition Commission

${ }^{19}$ Association for Media Research, http://aimc.es/ clients. It is also expected that the recent launch of $\mathrm{HBO}$ and Amazon Prime will increase the ratio of subscribers.

The aggressive campaigns by Spanish pay-TV operators, such as Movistar, Vodafone and Orange, offering their customers free Smart TVs with only access to their own payTV platforms, will also contribute to a wider adoption of Smart TVs and of hybrid TV media services. According to the latest available report of the Asociación para la Investigación de Medios de Comunicación ${ }^{19}$ (AIMC) (2016), over 21\% of Spanish consumers paid to watch online movies or series in 2015 , compared to $10.8 \%$ in 2014 , showing a clear rising trend. However, according to the previously mentioned CNMC report, $70.8 \%$ of Spanish consumers claimed not to be interested in paying for more TV channels, as they were happy with the FreeTo-Air (FTA) offer on DVB-T channels. Just over 30\% stated that the subscription cost was the reason behind not contracting such "premium" channels.

At the time of writing this paper, as far as authors know, in Spain, several DVB-T and radio channels have announced the availability of HbbTV services. Nevertheless, only RTVE ${ }^{20}$ (RTVE Red Button service), TV3 and EITB $^{21}$ are really providing them. The addition of other Spanish major broadcasters (e.g., Mediaset, A3Media..., with nearly $60 \%$ of share) to this standard would still be needed to definitively boost the use of hybrid TV media services.

Likewise, the number of companies offering new OTT products to provide premium, enriched and personalized services, and generating new revenues, is also growing in Spain, especially within the sports area. Mx1's platform ${ }^{22}$ is used by Football Club Barcelona for their Game Pass service ${ }^{23}$. RTVE brought viewers the 2016 Olympic Games through all possible consumption devices. Likewise, the International Olympic Committee launched a 24 hours per day OTT Olympic Channel.

\section{Demand of hybrid services for different content genres}

Leisure apps (e.g., for sports, games, films, reality shows, music...) could be expected to be the ones prompting the content production for hybrid and shared TV media services. Nevertheless, the study reveals that other types of genres, such as news, documentaries, science or educational programs, are also demanded by consumers. So they should be considered by content producers and providers when offering those services too.

On the one hand, according to Table 2, the demand of extrarelated content for non-entertainment genres, such as news and non-fiction, overtake the one for entertainment genres, such as sports and games. Moreover, male participants were significantly more interested in extra-related content for sports, games and porn genres, while female participants where a bit more interested in films, series and reality shows genres.

On the other hand, according to Table 8 , consumers showed

\footnotetext{
${ }^{20}$ Spanish Public TV.

21 TV3 and EITB are the Catalonian and Basque regional public TV broadcasters, respectively.

${ }^{22} \mathrm{http}: / /$ www.mx1.com/, former RR Media company

23 https://www.fcbgamepass.com/
} 
higher interest in sharing experiences for entertainment genres, such as sports, games and series. In this regard, male participants were quite more interested in shared experiences in news, non-fiction, sports, music and games genres, while female participants were a bit more interested in series and reality shows genres.

\section{Analyzed use cases}

Very few participants had previously participated in similar experiences to the analyzed use cases before (Table 3 ). This fact reflects the current low penetration of hybrid TV media services in Spain at the time the study took place. Only $24.7 \%, 8.5 \%$, $7.1 \%$ and $21.3 \%$ declared having had previous similar experiences to cases 1 to 4 , respectively. The previous experiences with the analyzed use cases were higher for male participants than for female participants, especially for use cases 1 and 3 (Table 3). Nevertheless, while many of the participants with previous experiences declared their sensations were not positive, most of them showed interest in them (90.7\%, 91.7\%, 79.4\% and 78.5\%, respectively) and thought that technology still had to be enhanced to successfully deploy those services (Figure 4). Except for use case 1, in which similar percentages of male and female participants were interested, for the other three use cases a higher percentage of male participants declared being interested in them.

Most of the participants identified many benefits (Table 6), clearly reflecting the potential of these services: more access to information, social integration, being able to interact with the content and other consumers, sharing experiences with remote consumers, having the possibility of personalizing the media consumption experience, and, in general, a more immersive and enjoyable experience. Social integration is a very important benefit that has been identified in responses related to use case 1 (e.g., foreign people being able to select the audio related to TV content in their native language; and hearing-impaired people being able to use headphones in their companion devices and adjust the audio level to their needs).

Nevertheless, participants also identified some limitations to overcome in order to successfully enjoy these services (Table 7). Among them, lack of sync, and low-speed connections were the most common ones.

In particular, lack of sync was identified as a drawback by $78.1,60.8,64.3$ and $55.9 \%$ of participants for use cases 1 to 4 , respectively. Media sync has been (and still is) a key research challenge since the advent of distributed media systems to guarantee a satisfactory QoE to the consumers. Although many media sync solutions have been proposed up to date ([30], [23]), they are mostly based on proprietary solutions, and they have been mostly focused on specific applications or use cases. Nevertheless, very few of them have tackled the problem of hybrid sync (e.g., [31], [32] and [33]) and, as far as authors know, they have not been extensively implemented in open large-scale services. Given the commercial interest in media sync and the disadvantages of proprietary technologies (e.g.

$24 \quad$ http://advanced-television.com/2015/10/12/spain-leads-european-ftthpenetration/ (last access: January 2017) fingerprinting and watermarking techniques [34] [35]), researchers, manufacturers, broadcasters and telecom operators have started developing a new wave of international standards ([36], [37]). Nevertheless, more work is still needed in that direction. For example, the latest release of the HbbTV standard (2.0.1 version) specifies functionalities and provides guidelines to achieve hybrid media delivery and consumption of broadcast and related broadband contents, on both single devices -MS and on different devices in multi-screen scenarios - one or various MS plus one or various $\mathrm{CS}-$, in a synchronized manner. However, this version of the standard does not provide fullfledged media sync solutions. It misses important aspects to successfully deploy hybrid TV media services. In addition, commercial platforms fully implementing this latest version of HbbTV are still unavailable.

Regarding the second main identified limitation, bandwidth insufficiency, it can be overcome by improving the communications infrastructures, technologies and networks. In this context, the major operators in Spain are increasing the deployment of their fiber optic networks and improving their mobile access networks. According to the Fiber-To-The Home (FTTH) European Council, in October 2015, Spain was the only EU country with over 10 million FTTH passed homes ${ }^{24}$. The latest data from CNMC (see footnote 17) reveal that FTTH deployment in Spain has grown by more than $50 \%$ over the last year, reaching more than 4 million of active lines against 1.57 million one year ago. Active lines, with speeds of up to or over $30 \mathrm{Mbps}$, also grew by $82 \%$ in a year. Despite these advances, the rise in the demand of high-quality (e.g., UHD and SUHD) media contents and the use of multi-party high-quality audio/video conferencing tools as an interaction channel will demand extra network resources (e.g., bandwidth). It will require additional solutions to overcome possible congestion and scalability problems.

Focusing on the analyzed use cases, regarding the multi-view camera use case, a pilot was carried out in Spain under the EU financed TV-RING project. It was about broadband multicamera services and was carried out in the Gurb municipality in the region of Catalonia (Spain) ${ }^{25}$. With more than 80 people distributed in 20 homes, the project conducted almost 20 activities. Contents such as TV shows, football and Barcelona's mayoral debate were selected. Among others, a relevant finding of that pilot was that hardware (HW) performance problems have a serious impact on the QoE. Delays originated due to the HW performance above 5-6s were found and caused frustration to users. Users considered delays above 8-10s unacceptable.

Another pilot was carried out in the Netherlands ${ }^{26}$ also under the same project, implementing three different services, one of them related to our use case 2 . That service investigated how an HbbTV app can act as a central interface for gaming between second screen devices in a household or home network. All the personal devices in a household were connected to a 'quizmaster' device. This idea stimulated competition and social interaction in a household, greatly enjoying the social

\footnotetext{
${ }^{25}$ http://www.tvring.eu/project/spanish-pilot/the-multicamera-service/ (last access: January 2017)

${ }^{26}$ http://www.tvring.eu/project/dutch-pilot/ (last access: January 2017)
} 
gaming experience around a TV show. The application created a clear benefit to linear TV. Participants did not feel that the second screen app distracted them from the show. They considered timely notifications that input from them would be required as very important. Other drawn conclusions were that tablets were the best-considered devices for second screen apps. When participants used laptops they reported to more easily open other applications and be distracted from the show. Besides, at least for participants alone in the room, it was interesting to receive comments by participants in other households. In that case, this means allowing participants to view why they were for or against a certain statement in the show.

Regarding the Social TV use case, special attention should be paid to the use of Social Media and other interaction channels between consumers. In this context, many consumers use offline communications, by text messaging other consumers about what they are watching on TV. They offer them a more private/personal means of communication. According to Table 4, there were no significant differences between the participants' preferences regarding the use of integrated (ad-hoc) or independent (but most popular) social interaction tools (both around 27\%), such as Skype, Twitter or WhatsApp. Social Media as an interaction channel (e.g., by using official accounts and/or associated hashtags in Twitter) has the potential of providing more compelling TV experiences. Indeed, the study shows that participants used Social Media to have a more enjoyable and personalized experience and to be aware about what other consumers are posting about TV events. A related study from the Connected Home UX (CHX) group at Strategy Analytics ${ }^{27}$, in 2015 (focused on US and UK participants), found that while the use of Social Media is high, the use in relation to what is on TV is low. Participants found Social TV concepts more suitable for a personal device and individual, rather than for a collective, watching experience.

Social TV has the potential to change the way in which consumers interact with Social Media, in relation to what they watch on TV. Nevertheless, certain features must be enhanced, such as, for example, to make the use and navigation on the big screen easier, to provide a collective TV experience, and to be customizable. While many participants found the use of Social Media appealing, some of them were also apprehensive about their suitability on the big screen, concerned that it may provide a distracting and intrusive TV experience.

Another pilot related to the Social TV use case was carried out in Germany also under the TV-RING project. It was focused on providing multimedia content on complementary channels (TV, website, HbbTV and Social Media) to a young audience ${ }^{28}$. Young users were not so much looking for extra information, but preferred it as video content. Related extra content should be visible, and should be preferably video. Involving users in communication about a program through a Social Media feed on TV (i.e. Social TV) worked best when "celebrities" (i.e., actors from the series or experts) joined the chat discussion via their Twitter or Facebook channels. In that case, viewers were more easily tempted to participate.

Other studies have investigated other aspects regarding the Social TV use case, such as, e.g., [38], [39], [40], [41], [42], [13] and [16]. All of them hinted in the direction that hybrid sync is very important for a good QoE in shared TV watching, as communication patterns could be broken if it is not provided. In [38], a practical study was presented comparing watching TV together in two distributed groups with watching TV in one large group. Distributed group TV watching shared many similarities with co-located TV watching. In both cases, conversations evolved mainly around program contents and occur at silent periods. Also, the amount of conversation was similar, and visual interaction was limited. In [39], a larger inhome field trial for Social TV was conducted, obtaining similar conclusions. Data from 50 households without Social TV functionality were compared with data from households with that functionality. It showed that Social TV users felt more connected. Those results were also confirmed for friends and strangers through tests in a controlled environment in [40]. The study in [41] claimed that IDMS helps users to feel closer and more connected when watching video together, while apart. In [42], Motorola performed an early experiment to investigate the benefits of shared distributed TV watching. Friends living in five homes got installed a Social TV app, on a provided device, allowing them to have voice and text interaction channels while watching TV. Multiple interviews with the participants were conducted to determine the benefits on the QoE. One of the main conclusions drawn was that the bond between the friends had strengthened by the end of the study and that they felt more connected to each other. In [13], an experiment with 36 participants was conducted in order to determine the allowable asynchrony levels between receivers in a Social TV scenario. The participants watched a quiz show, which is a very sociable genre according to [43] and [44]. It found that, when using voice chat, users notice synchronization differences sooner, are more annoyed and feel more together than when using text chat. However, users with high text chat activity notice sync differences similarly than participants using voice chat. It also concluded that a direct communication link between users watching video together is desirable and increases the level of 'togetherness'. In [16], a controlled experiment simulating the shared football watching experience was conducted. 18 users participated, watching clips in different rooms, while sharing a voice interaction channel. Empirical evidence showed that relative delays encountered in digital TV degrade the football watching experience. Especially large differences were noticed quickly by participants and are a plausible reason to change the content provider. The allowable asynchrony thresholds in that scenario were also evaluated, with similar results than in [13].

All the aforementioned research studies have shown the convenience and benefits of hybrid TV media services. They have provided initial insights about the influence of delays and its variability and the relevance of media sync. However, they do not provide insights about personal preferences, needs or opinions about the service, as our study does. 


\section{E. Opportunities for media researchers, developers and market stakeholders}

The use of hybrid delivery technologies brings many opportunities to offer new fascinating services and to improve user experience (e.g., customization, interactivity/bidirectionality, new formats, adaptability, ubiquity, cost savings...). Nevertheless, more efforts are still needed to overcome key remaining challenges, such as compatibility guarantees, standard compliance, hybrid sync, performance improvement, etc.

The latest version release of the HbbTV standard has represented a big step towards the adoption of hybrid TV media services. However, it still lacks full-fledged solutions to successfully deploy these kinds of services. Some examples of remaining challenges that need to be addressed are: 1) signaling mechanisms to discover, associate and describe the related media contents; 2) interaction and negotiation mechanisms between the available devices; and 3) adaptive media sync solutions (including protocols, algorithms and adjustment techniques) to accurately time-align the consumption of the related contents.

The main goal is to achieve a seamless convergence, coordination and inter-operability between the available delivery technologies. Thus, leveraging their strengths and complementary characteristics, in order to no longer conceive them as isolated worlds, but instead as a unique hybrid media ecosystem. In addition, a user-transparent interaction and coordination between the available (multi-)connected devices for media consumption is desirable. This will extend the media consumption possibilities and will provide a new wave of enriched services, opening the door to new business models.

Most of the participants in the present study agreed that better or improved technologies are still needed to successfully provide services like the analyzed use cases (Figure 4). Further research is required to solve the identified drawbacks (new technologies, standards, media sync solutions, better applications...). This will contribute to maximize the QoE perceived by consumers and, thus, to increase the chances of successful deployment and acceptability of hybrid services.

Apart from the above technological aspects, the current usability of Smart TVs in Spain is not as good as expected ${ }^{29}$. The ease of use, the user interface, responsiveness, customization options, the compatibility with other ecosystems (standard compliancy) and the available apps/services/features need to be improved. In these aspects, nowadays, companion devices are already surpassing Smart TVs. Likewise, consumers are turning into the usage of multiple consumption devices. According to the obtained results, $36.5 \%$ of consumers interested in extra(-related) contents still preferred to watch them on the same screen, $22.9 \%$ on a secondary screen, and the rest did not mind whatever screen the contents were presented on (Section IV.D). The emerging multi-screen ecosystem brings many design opportunities for developers, such as creating second screen apps as companions to the TV program, or multi-screen apps to consume media content on the many available devices and screens, working as a whole, and overcoming inter-operability problems. However, these applications must be carefully designed, so that they will not be considered distracting, stressful or intrusive. Developers should also continue integrating new features to account for evolving Social TV behaviors, as TV networks themselves are utilizing such behaviors to their benefit (e.g., advertising).

In addition, more initiatives from content producers are needed to change the way in which consumers interact with Social Media in relation to what they watch on TV (e.g., bringing Social TV to the TV itself, rather than designating such behaviors to personal devices).

Finally, some studies have been focused on determining the acceptable asynchrony ranges that are tolerable to the human perception regarding different use cases in which media sync is needed (Section III). However, these studies were mostly focused on intra-stream sync (tolerance to jitter) and on interstream sync (tolerance to lip-sync). Although some preliminary experiments for IDES and IDMS have been conducted, they need to be extended in settings that are more realistic and for other use cases, apart from Social TV. Moreover, the allowable limits for the different hybrid TV media services have not been rigorously determined yet. Therefore, more QoE tests will be needed to find them out. Such limits should be obtained through exhaustive research and very rigorous objective and subjective assessments, possibly including longer-term testing in live systems, in contrast to testing in artificial test environments. Likewise, such experiments should be conducted for each particular use case under study, as the ranges of tolerable asynchrony levels strongly depend on the usage scenario. Having accurate references for such limits will be very relevant for network operators, content providers and developers to put their efforts on not exceeding them. Otherwise, it would lead to users' noticeability and, even worse, annoyance and frustration (with consequent complaints and very likely un-subscriptions to the service).

Combined, all these aspects and research efforts will definitely contribute to improve the hybrid TV media experience and value of multi-screen applications.

\section{CONCLUSIONS}

The daily use of digital technologies and services seemed to be only exclusive for young people in the recent past. They have been incorporating them in their everyday activities, shaping a real-time connected lifestyle in which personalized media experiences are demanded. Nevertheless, digitalization is progressively reaching all ages and social spheres through hybrid technologies and services.

In this paper, the concept of hybrid TV media services has been introduced, and illustrated with some representative examples. The need for different types of media sync (hybrid sync) as a key technological enabler for these services has also been emphasized and justified. Moreover, the results of a

\footnotetext{
29 This also happens in Europe, as corroborated in the last HbbTV Symposium in October 2016.
} 
research study conducted in Spain have been presented and discussed. This research has been focused on collecting the habits, demands, opinions, preferences and expectations of more than 1000 Spanish users regarding four representative hybrid TV media consumption use cases.

From the obtained results, it can be concluded that hybrid TV media services are not widely and successfully deployed in Spain yet. Nevertheless, it is expected that they will have a tremendous impact on the upcoming media consumption landscape. Therefore, it is convenient to continue research in this field by devising proper standard-compliant technological solutions and equipment, by producing appropriate contents, and by deploying proper services and applications.

The presented results are expected to be very useful to drive new research efforts in this direction. Moreover, they can also be very valuable and encouraging for all media market stakeholders (not only in Spain, but also in Europe and worldwide). The results will help to determine what should be implemented, offered and/or enhanced in the near future regarding media content production, delivery and consumption processes as well as to overcome the identified challenges and limitations. It will contribute to provide better QoE to the consumers and increase the market adoption of these services.

As future work, the study could be extended to other countries. Likewise, it could also be interesting to identify and classify different consumer profiles (e.g., based on their age, sex, habits and interests). Finally, authors are very interested in conducting the QoE tests mentioned in Section V, focused on determining the allowable asynchrony limits in different emerging hybrid TV media services in which hybrid sync plays a key role.

\section{ACKNOWLEDGMENT}

The authors would also like to thank Anne Moeskops, Isaac Almendros, Rebeca Díez, Fco. Javier Pastor, Dani Palacio, Francisco de Zulueta and Vicente Domingo Struch, for their invaluable help during the study.

\section{REFERENCES}

[1] F. Boronat, M. Montagut, H. Stokking, O. Niamut, "The need for InterDestination Synchronization for Emerging Social Interactive Multimedia Applications", IEEE Communications Magazine, November 2012, pp. $150-158$.

[2] C. Hesselman et al., "Sharing enriched multimedia experiences across heterogeneous network infrastructures", in IEEE Communications Magazine, vol. 48, no. 6, pp. 54-65, June 2010. doi: 10.1109/MCOM.2010.5473865

[3] M. Wijnants, W. Lamotte, J. De Meulenaere, W. Van den Broeck, "Qualitative Assessment of Contemporary Media Sharing Practices and Their Relationship to the sMS Platform", Hasselt University, IBBTSMIT, pp. 31-36, 2012.

[4] Hybrid Broadcast Broadband TV standard. http://www.hbbtv.org/

[5] Advanced Television Systems Committee. http://atsc.org

[6] ISO/IEC 23008-1: 2014. "Information Technology. High Efficiency Coding and Media Delivery in Heterogeneous Environments. Part 1: MPEG Media Transport (MMT)".

[7] I. Vaishnavi, P. Cesar, D. Bulterman, O. Friedrich, S. Gunkel, D. Geerts, "From IPTV to Synchronous Shared Experiences Challenges in Design: Distributed Media Synchronization," Sig. Processing: Image Commun. vol. 26, no. 7, pp. 370-77, August 2011.

[8] R. Stanton, "Second Screen Revolutionizing the Television Experience", TATA Consultancy Services, White Paper, 2012,
http://www.tcs.com/SiteCollectionDocuments/White\%20Papers/Consult ing Whitepaper_Second-Screen-Revolutionizing-TelevisionExperience-Part-1 0512-2.pdf (last access: January 2017).

[9] YUME (in Spanish), "La etnia emergente: interacción de los consumidores hispanos en todas las pantallas", White Paper, 2014, http://www.yume.com/es/node/627? filename=YuMe US Hispanic Con sumer_Interactions_Across_Screens.pdf\&lval=advres\&pge $=$ res $\quad$ (last access: January 2017).

[10] H. Schulzrinne, S. Casner, R. Frederick, and V. Jacobson, "RTP: A Transport Protocol for Real-Time Applications. IETF Internet Standard, RFC 3550, July 2003.

[11] M. Montagud, F. Boronat, H. Stokking, R. van Brandenburg, "Interdestination multimedia synchronization: schemes, use cases and standardization", Springer-Verlag, Multimedia Systems, pp. 459-482, 10 July 2012.

[12] R.N. Mekuria, "Inter-destination media synchronization for TV broadcasts", Master Thesis, Faculty of Electrical Engineering, Mathematics and Computer Science, Department of Network architecture and Services, Delft University of Technology (2011).

[13] D. Geerts, I. Vaishnavi, R. Mekuria, M.O. Van Deventer, P. Cesar, "Are we in sync?: synchronization requirements for watching on-line video together", CHI '11, New York, USA (2011)

[14] F. Boronat, R. Mekuria, M. Montagud, and P. Cesar, "Distributed media synchronization for shared video watching: Issues, challenges, and examples," in Social Media Retrieval, N. Ramzan, R. van Zwol, J.-S. Lee, K. Clüver, and X.-S.Hua, Eds. ser. Springer Computer Communications and Networks Series. London, U.K.: Springer-Verlag, 2013, pp. 393-431.

[15] W. Kooij, H. Stokking, R. van Brandenburg, P-T de Boer, "Playout delay of TV signals: measurement system design, validation and results", ACM TVX 2014, Newcastle (UK), June 2014.

[16] R. Mekuria, P. Cesar, and D. Bulterman. 2012. "Digital TV: the effect of delay when watching football". In Proceedings of the 10th European conference on Interactive tv and video (EuroiTV '12). ACM, New York, NY, USA, 71-74. DOI=http://dx.doi.org/10.1145/2325616.2325632

[17] ITU-T G.1050 Recommendation, "Network model for evaluating multimedia transmission performance over internet protocol", 2007.

[18] ITU-T Rec. G. 114: "One-way transmission time", 2003.

[19] J. Jansen, P. Cesar, D.C.A. Bulterman, T. Stevens, I. Kegel, J. Issing, "Enabling Composition-Based Video-Conferencing for the Home", IEEE Transactions on Multimedia (TMM), 13(5), pp. 869-881, October 2011.

[20] M.O. Van Deventer, H. Stokking, O.A. Niamut, F.A. Walraven, V.B. Klos, "Advanced Interactive Television Service Require Synchronization”, IWSSIP 2008. Bratislava, June (2008).

[21] L. Beloqui, F. Boronat, M. Montagud and H. Melvin, "Understanding Timelines within MPEG Standards", IEEE Communications Surveys and Tutorials journal, ISSN: 1553-877X, 18(1): pp. 368-400 (2016).

[22] M. Montagud, F. Boronat, "Enhanced adaptive RTCP-based InterDestination Multimedia Synchronization approach for distributed applications", Computer Networks, Volume 56, Issue 12, 16 August 2012, Pages 2912-2933, ISSN 1389-1286, http://dx.doi.org/10.1016/j.comnet.2012.05.003.

[23] Z. Huang, K. Nahrstedt, R. Steinmetz. 2013. "Evolution of temporal multimedia synchronization principles: A historical viewpoint”. ACM Trans. Multimedia Comput. Commun. Appl. 9, 1s, Article 34 (October 2013), 23 pages. DOI=http://dx.doi.org/10.1145/2490821

[24] R. Steinmetz, "Human perception of jitter and media synchronization", IEEE J. Sel. Areas Commun. 14(1), 61-72 (1996)

[25] B. Rainer, S. Petscharnig, C. Timmerer, and H. Hellwagner, "Is one second enough? evaluating $\mathrm{QoE}$ for inter-destination multimedia synchronization using human computation and crowdsourcing", in Quality of Multimedia Experience (QoMEX), 2015 Seventh International Workshop on, pages 1-6. IEEE, 2015.

[26] Y. Bang et al., "Wireless network synchronization for multichannel multimedia services," in Proc. 11th ICAT, Feb. 2009, vol. 2, pp. 10731077.

[27] The Office of Research Integrity (ORI), "Descriptive study design", available at: http://ori.hhs.gov/education/products/sdsu/res des1.htm (last access: January 2017).

[28] A. Bryman, "Social research methods", 2012, ed. Oxford University Press, 4th edition, 808 pages, ISBN 978-0-19-958805-3.

[29] J. Boyny, "HbbTV and the Connected Consumer. Where Are We Today?", HbbTV Symposium, Madrid, October 18-19, 2016.

[30] F. Boronat, J. Lloret, M. Garcia, "Multimedia group and inter-stream synchronization techniques: A comparative study", Information Systems, Elsevier, Volume 34, Issue 1, Pages 108-131, March 2009. 
[31] C. Concolato, S. Thomas, R. Bouqueau, J. Le Feuvre, "Synchronized Delivery of Multimedia Content over Uncoordinated Broadcast Broadband Networks", ACM MMSys'12, February 22-24, 2012, Chapel Hill, North Carolina, USA.

[32] L. Beloqui, P. O Flaithearta, H. Melvin, "Interactive Multi-source Media Synchronization for HbbTV", Media Synchronization Workshop 2012, October 11, 2012, Berlin, Germany.

[33] J. Le Feuvre, V-T-T NGuyen, W. Hammidouche, P. Marchal, R. Monnier, P. Dupain "A Test Bed for Hybrid Broadcast Broadband Services", Media Synchronization Workshop 2015, June 3, 2015, Brussels, Belgium.

[34] A.J. Mason, "EBU tests of commercial audio watermarking systems", BBC R\&D Whitepaper 101, December 2004 http://www.bbc.co.uk/rd/publications/whitepaper101 (last access: January 2017).

[35] R. Bardeli, J. Schwenninger and D. Stein, "Audio Fingerprinting for Media Synchronisation and Duplicate Detection", MediaSync Workshop, in conjuntion with ICIN 2012 (International Conference on Intelligence in Next Generation Networks), Berlin (Germany), 2012 (8-11 October)

[36] R. Van Brandenburg, H. Stokking, M. O. Van Deventer Van, F. Boronat, M. Montagud, K. Gross, "RTCP for inter-destination media synchronization", RFC 7272, June 2014.

[37] O. van Deventer, H. Stokking, M. Hammond and P. Cesar, "Standards for multi-stream and multi-device media synchronization", in IEEE Communications Magazine, vol. 54, no. 3, pp. 16-21, March 2016.

[38] L. Oehlberg, N. Duchenaut and J. Thornton, "Social TV: designing for distributed, sociable television viewing", in: Proceedings of EuroITV, Athens, Greece (2006)

[39] E. Boertjes, et al., "ConnecTV: share the experience", in: Proceedings of EuroITV, Amsterdam, the Netherlands, pp. 139-140 (2007).

[40] D. Weisz, et al., "Watching together: integrating text chat with video", in: CHI '07: Proceedings of the SIGCHI Conference on Human Factors in Computing Systems (2007). doi:10.1145/1240624.1240756.

[41] D. A. Shamma, M. Bastea-Forte, N. Joubert, and Y. Liu. 2008. Enhancing online personal connections through the synchronized sharing of online video. In CHI '08 Extended Abstracts on Human Factors in Computing Systems (CHI EA '08). ACM, New York, NY, USA, 2931-2936. DOI=http://dx.doi.org/10.1145/1358628.1358786

[42] E. Huang, et al., "Of social television comes home: a field study of communication choices and practices in TV-based text and voice chat", in: CHI '09: Proceedings of the SIGCHI Conference on Human Factors in Computing Systems (2009). doi:10.1145/1518701.1518792

[43] D. Geerts, P. Cesar, D. Bulterman, "The implications of program genres for the design of social television systems", 1st international conference on Designing interactive user experiences for TV and video (UXTV'08), Silicon Valley, California (USA), October 2008.

[44] D. Geerts, D. De Grooff, "Supporting the social uses of television: sociability heuristics for social TV", ACM CHI Conference on Human Factors in Computing Systems, CHI 2009, pp. 595-604, Boston (USA), April 2009.

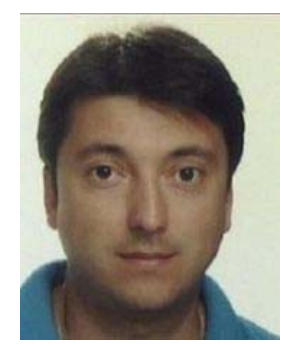

Fernando Boronat (M'93-SM'11), was born in Gandia (Spain), and went to the Polytechnic University of Valencia (UPV) in Spain, where he studied Telecommunications Engineering. $\mathrm{He}$ received the M.E. and Ph.D. degrees in telecommunication engineering from the UPV in 1994 and 2014, respectively. After working for several Spanish telecommunication companies, he moved back to the UPV in 1996. Currently he is an Assistant Professor in the Communications Department. Dr. Boronat is the Head of the Immersive Interactive Media R\&D Group (http://iim.webs.upv.es) at the Gandia Campus of UPV. His main topics of interest are communication networks, multimedia systems, multimedia protocols, and media synchronization. He is the author of two books, several book chapters, an IETF RFC and more than 100 research papers. He is Editor of "MediaSync: Handbook on Multimedia Synchronization" (Springer, 2017). He is involved in several IPCs of national and international journals and conferences. He is member of IEEE and ACM.

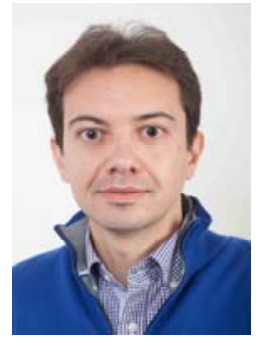

Mario Montagud was born in Montitxelvo (Spain). $\mathrm{He}$ received a $\mathrm{BsC}$ in Telecommunications Engineering in 2011, an MsC degree in "Telecommunication Technologies, Systems and Networks" in 2012 and a PhD degree in Telecommunications (Cum Laude Distinction) in 2015, all of them at the Polytechnic University of Valencia (UPV, Spain). During his PhD degree and after completing it, he did 3 research stays (18 months) at CWI (The National Research Institute for Mathematics and Computer Science in the Netherlands) in Amsterdam. He also has experience as postdoctoral researcher at UPV. His topics of interest include Computer Networks, Interactive and Immersive Media, Synchronization, Human Computer Interaction (HCI) and QoE. Mario is (co-) author of over 50 scientific and teaching publications, and has contributed to standardization within the IETF. He is TPC member of several international conferences, coorganizer of the international MediaSync Workshop series, member of the Editorial Board of international journals, and Communication Ambassador of ACM SIGCHI. He is also Editor of "MediaSync: Handbook on Multimedia Synchronization" (Springer, 2017). Website: https://sites.google.com/site/mamontor/

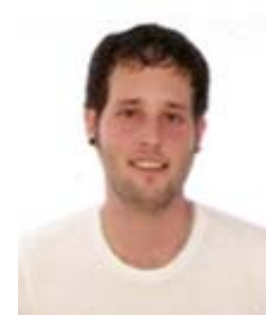

Dani Marfil was born in Gandia (Spain) and went to the UPV in Spain, where he studied Informatics Technical Engineering (2011), Telecommunications Degree and Telecommunication Technologies (2015), Systems and Networks Msc (2016). He is a $\mathrm{PhD}$ student and an assistant researcher and developer in the Immersive Interactive Media R\&D Group. His main topics of interest are communication networks, code developing and media synchronization. He is the author of one book chapter and four papers.

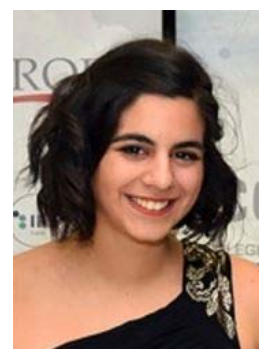

Clara Luzón was born in Hellín (Spain). She finished a Bachelor's Degree in Telecommunications Systems, Sound and Image Engineering in 2016, in the UPV, in Spain. Now she is studying Artificial Intelligence, Pattern Recognition and Digital Imaging MsC also at UPV. She is collaborating as developer in the Immersive Interactive Media R\&D Group at the Gandia Campus of UPV. Her main topics of interest are artificial intelligence, multimedia systems and media synchronization. She is the author of three papers and has received several academic awards. 